\title{
SAK Principle for a class of Grushin-type operators
}

\section{Lidia Maniccia and Marco Mughetti}

\begin{abstract}
We prove Fefferman's SAK Principle for a class of hypoelliptic operators on $\mathbb{R}^{2}$ whose nonnegative symbol vanishes anisotropically on the characteristic manifold.
\end{abstract}

\section{Introduction}

In his celebrated paper [5], Fefferman suggests a strategy to get a priori estimates (SAK principle). Suppose that one wants to know whether a given estimate of the form

$$
C\left\|q^{w} u\right\|_{0} \leq\left\|L^{w} u\right\|_{0}+\text { "small error terms", } \quad \forall u \in \mathcal{S}\left(\mathbb{R}^{n}\right),
$$

holds for given symbols $L, q \in S^{2}\left(\mathbb{R}^{n}\right)$. Here $S^{m}\left(\mathbb{R}^{n}\right), m \in \mathbb{R}$, is the set of "global symbols" $a(x, \xi)$ satisfying $\left|\partial_{x}^{\alpha} \partial_{\xi}^{\beta} a(x, \xi)\right| \leq C_{\alpha, \beta}(1+|\xi|)^{m-|\beta|}$ for all $(x, \xi) \in \mathbb{R}^{2 n}$ and all $\alpha, \beta \in \mathbb{Z}_{+}^{n}$, and $a^{w}$ denotes the Weyl quantization of $a$ (see [10]). Fefferman's idea is to obtain (1.1) by a comparison of the symbols $L$ and $q$. This is in analogy to the Fefferman-Phong inequality proved in [4]:

$$
S^{2}\left(\mathbb{R}^{n}\right) \ni a \geq 0 \Longrightarrow\left(a^{w} u, u\right)_{L^{2}} \geq-C\|u\|_{0}^{2}, \quad \forall u \in \mathcal{S}\left(\mathbb{R}^{n}\right) .
$$

Hérau proved (1.1) for $n=1$ assuming that $L(x, \xi) \geq 0$ and $|q(x, \xi)| \leq$ $L(x, \xi)$ for every $(x, \xi) \in \mathbb{R}^{2}$ (see [8]). Actually, Fefferman conjectured that (1.1) holds under a weaker assumption, namely

$$
\max _{\mathcal{B}_{\mu}}|q(x, \xi)| \leq c \max _{\mathcal{B}_{\mu}} L(x, \xi),
$$

where $\left\{B_{\mu}\right\}_{\mu \in \mathbb{N}}$ is a suitable "partition" of the phase space $\mathbb{R}_{x}^{n} \times \mathbb{R}_{\xi}^{n}$ associated with $L(x, \xi)$.

2000 Mathematics Subject Classification: 35B45, 35S05.

Keywords: SAK principle, a priori estimates, Grushin operators, Weyl-Hörmander calculus. 
In this paper, we prove this result in the case $n=2$ and $L^{w}$ being the Grushin operator with symbol

$$
L(x, \xi)=\xi_{1}^{2}+x_{1}^{2 h} \xi_{2}^{2}, \quad(x, \xi)=\left(x_{1}, x_{2}, \xi_{1}, \xi_{2}\right) \in \mathbb{R}^{4}, h \in \mathbb{Z}_{+} .
$$

Similar arguments work in higher dimension for polynomial symbols having the same kind of anisotropic vanishing. More precisely, if $x=\left(x^{\prime}, x^{\prime \prime}\right) \in$ $\mathbb{R}^{\mu} \times \mathbb{R}^{n-\mu}$ (accordingly $\xi=\left(\xi^{\prime}, \xi^{\prime \prime}\right)$ ), one can consider operators having real homogeneous polynomial symbols that near the characteristic manifold, $\left\{(x, \xi)=\left(x^{\prime}, x^{\prime \prime}, \xi^{\prime}, \xi^{\prime \prime}\right) \in \mathbb{R}^{2 n}: x^{\prime}=\xi^{\prime}=0\right\}$, behave like $|\xi|^{2}\left(\left|x^{\prime}\right|^{2 h}+\right.$ $\left.\left|\xi^{\prime}\right|^{2} /|\xi|^{2}\right)$. The required adjustments to treat these cases can be easily deduced and are here omitted. It is worthwhile to observe that the Grushin operator in $\mathbb{R}^{2}$ is "meaningful" in view of reduction theorems given in [5].

Before stating the precise result, let us observe that the weaker assumption (1.2) on the symbols has, in general, non trivial consequences. For example, considering real vector fields $X_{j}$ with Weyl symbols $p_{j}$, a pointwise comparison of the symbols yields

$$
\left\|X_{j}^{2} u\right\|_{0} \leq C\left(\left\|\sum_{j} X_{j}^{2} u\right\|_{0}+\|u\|_{\epsilon}\right) .
$$

In fact, we have $\sigma^{w}\left(X_{k}^{2}\right)=p_{k}^{2}+i\left\{p_{k}, p_{k}\right\} / 2+S^{0}=p_{k}^{2}+S^{0}, p_{k}^{2}(x, \xi) \leq$ $\sum_{j} p_{j}^{2}(x, \xi)$. The full SAK Principle would even imply that

$$
\left\|X_{j} X_{k} u\right\|_{0} \leq C\left(\left\|\sum_{j} X_{j}^{2} u\right\|_{0}+\|u\|_{\epsilon}\right)
$$

since the symbol of $X_{j} X_{k}$ is $p_{j} p_{k}+i\left\{p_{j}, p_{k}\right\} / 2+S^{0}$ and it satisfies (1.2) for suitable rectangles $B_{\mu}$. In fact, $\left|p_{j} p_{k}\right| \leq c \sum_{j} p_{j}^{2}$ but a pointwise comparison of $\left\{p_{j}, p_{k}\right\}$ by the symbol of $\sum_{j} X_{j}^{2}$ is in general not possible as one easily checks in our case $p_{1}=\xi_{1}$ and $p_{2}=x_{1}^{h} \xi_{2}$.

Some preliminary facts are needed in order to state the main Theorem of this paper. From (1.3), it is easily seen that $L(x, \xi) \geq 0$ with characteristic manifold given by

$$
\Sigma=\left\{\left(x_{1}, x_{2}, \xi_{1}, \xi_{2}\right) \in \mathbb{R}^{4}: x_{1}=\xi_{1}=0, \xi_{2} \neq 0\right\} .
$$

Definition 1.1 Let $R>0$ be a suitable large fixed constant and consider, for $(x, \xi) \in \mathbb{R}^{4}$,

$$
D_{\Sigma}(x, \xi)=\left(\frac{x_{1}^{2 h}}{\left(1+x_{1}^{2}\right)^{h}}+\frac{\xi_{1}^{2}}{R^{2}+|\xi|^{2}}+\frac{R^{2 h /(h+1)}}{\left(R^{2}+|\xi|^{2}\right)^{h /(h+1)}}\right)^{1 / 2} .
$$

We then define, for every $(x, \xi),(y, \eta) \in \mathbb{R}^{4}$, the Hörmander metric

$$
G_{(x, \xi)}(y, \eta)=\frac{|y|^{2}}{D_{\Sigma}(x, \xi)^{2 / h}}+\frac{|\eta|^{2}}{\left(R^{2}+|\xi|^{2}\right) D_{\Sigma}(x, \xi)^{2}} .
$$


We want to point out that the metric $G$ here considered is obtained by slightly modifying the metric used in [12] for the parametrix construction. As a consequence, the Weyl calculus defined by $G$ is strictly related with Boutet de Monvel's calculus (see [3]). It is worth to observe that, near the characteristic manifold $\Sigma$, the metric $G$ is equivalent to a metric defined only through the symbol $L$, namely

$$
G_{(x, \xi)}^{L}(y, \eta)=\frac{|y|^{2}}{\langle\xi\rangle^{-2 / h}\left(L(x, \xi)+\langle\xi\rangle^{2 /(h+1)}\right)^{1 / h}}+\frac{|\eta|^{2}}{L(x, \xi)+\langle\xi\rangle^{2 /(h+1)}},
$$

for $(x, \xi),(y, \eta) \in \mathbb{R}^{4}$. In the following Theorem, we denote by $c_{S V}$ the positive constant related to the slowly varying property of the metric $G$ in Definition 1.1:

$$
G_{X}(Y-X) \leq c_{S V} \quad \Longrightarrow \quad c_{S V} G_{Y}(Z) \leq G_{X}(Z) \leq \frac{1}{c_{S V}} G_{Y}(Z),
$$

$\forall X, Y, Z \in \mathbb{R}^{2} \times \mathbb{R}^{2}$.

Theorem 1.2 Let $q \in S^{2}\left(\mathbb{R}^{2}\right)$ and suppose that there exists a constant $0<$ $r \leq c_{S V}$ and a covering $\left\{B_{\left(x_{j}, \xi_{j}\right)}^{G}(r)\right\}_{j \in \mathbb{N}}$ of $\mathbb{R}_{x}^{2} \times \mathbb{R}_{\xi}^{2}$ such that

$$
\max _{B_{\left(x_{j}, \xi_{j}\right)}^{G}(r)}|q| \leq c \max _{B_{\left(x_{j}, \xi_{j}\right)}^{G}(r)} L, \quad \forall j \in \mathbb{N}
$$

where

$$
B_{\left(x_{j}, \xi_{j}\right)}^{G}(r)=\left\{(y, \eta) \in \mathbb{R}^{4}: G_{\left(x_{j}, \xi_{j}\right)}\left(y-x_{j}, \eta-\xi_{j}\right) \leq r^{2}\right\}
$$

and $c$ is a constant independent of $j \in \mathbb{N}$.

Then, for every compact set $K \subset \mathbb{R}^{2}$, there exists a positive constant $C=C(K)$ such that

$$
\|L(x, D) u\|_{0}^{2}+\|u\|_{0}^{2} \geq C\left\|q^{w} u\right\|_{0}^{2}, \quad \forall u \in C_{0}^{\infty}(K) .
$$

Note that one cannot expect to have (1.7) uniformly for all $u$ in $\mathcal{S}\left(\mathbb{R}^{2}\right)$, since $L$ is not globally bounded in $\mathbb{R}^{4}$.

It is worth pointing out that the decomposition of the phase space $T^{*} \mathbb{R}^{2}$ by means of the balls $B_{\left(x_{j}, \xi_{j}\right)}^{G}(r)$ given above is different from the one proposed by Fefferman in [6]. This shows that there is no a-priori uniqueness in the choice of the "good" partition of $T^{*} \mathbb{R}^{n}$. A very difficult problem is to understand what a "natural" definition of ball associated with a second order pseudodifferential operator is. This problem is studied in [13], where a sharp definition of subunit ball for non-negative symbols of subelliptic operators is discussed. 
The paper is organized as follows. In Section 2 we show that Theorem 1.2 can be "microlocalized" to Theorem 2.4 below and we develop some tools needed in Section 3 for the proof of Theorem 2.4. In the Appendix some technical results required throughout the paper are proved.

Acknowledgements. We thank A. Parmeggiani for introducing us to this argument and for the constant support.

\section{First and second level microlocalizations}

Definition 2.1 Let $K \subset \mathbb{R}^{2}$ be an arbitrary fixed compact set and let $\chi$ be a function in $C_{0}^{\infty}\left(\mathbb{R}^{2}\right)$ such that $\chi=1$ on a neighborhood of $K$ and $0 \leq \chi(x) \leq 1$. Define

$$
p(x, \xi)=\xi_{1}^{2}+\left[\chi(x) x_{1}^{2 h}+(1-\chi(x)) \frac{x_{1}^{2 h}}{\left(1+x_{1}^{2}\right)^{h}}\right] \xi_{2}^{2} .
$$

The symbol $p$ is an extension of the Grushin operator outside the compact set $K$, whose properties are stated in the lemma below.

Lemma 2.2 The following properties hold:

1) $p \in S^{2}\left(\mathbb{R}^{2}\right)$;

2) $p(x, \xi) \geq 0, \forall(x, \xi) \in \mathbb{R}_{x}^{2} \times\left(\mathbb{R}_{\xi}^{2} \backslash\{0\}\right)$, and $p(x, \xi)=0$ iff $(x, \xi) \in \Sigma$;

3) for every constant $C>0$ and for every (closed) rectangle $Q \subseteq \mathbb{R}^{4}$ such that $\Pi_{x}(Q) \subseteq\left\{\left(x_{1}, x_{2}\right) \in \mathbb{R}^{2}:\left|x_{1}\right| \leq C\right\}$ (here $\Pi_{x}$ is the projection $\left.(x, \xi) \in \mathbb{R}^{4} \mapsto x \in \mathbb{R}^{2}\right)$ one has

$$
\max _{Q} p(x, \xi) \sim \max _{Q} L(x, \xi) \text { uniformly in } Q,
$$

with $L$ defined by (1.3);

4) if $q \in S^{2}\left(\mathbb{R}^{2}\right)$ is such that

$$
\left\|p^{w} u\right\|_{0}^{2}+\|u\|_{0}^{2} \geq c\left\|q^{w} u\right\|_{0}^{2}, \quad \forall u \in \mathcal{S}\left(\mathbb{R}^{2}\right)
$$

for some positive constant c, then estimate (1.7) holds.

The previous lemma reduces the proof of Theorem 1.2 to the proof, under the same assumptions, of (2.1). We further want to give a microlocal version of (2.1). In order to do this we need to introduce a partition of $\mathbb{R}^{4}$ in suitable rectangles having sides parallel to the coordinate axes.

For any $d>0$ and for any $\bar{y}=\left(\bar{y}_{1}, \bar{y}_{2}\right) \in \mathbb{R}^{2}$, denote by $I_{d}(\bar{y})$ the rectangle $I_{d}(\bar{y})=\left\{y \in \mathbb{R}^{2}:\left|y_{i}-\bar{y}_{i}\right| \leq d /(2 \sqrt{2}), i=1,2\right\}$ (d is referred to as the diameter of $I_{d}(\bar{y})$ ), and consider a partition $\left\{Q_{\mu}^{1}\right\}_{\mu \in \mathbb{N}}$ of $\mathbb{R}^{4}$ with $Q_{\mu}^{1}=I_{1}\left(x_{\mu}\right) \times I_{M_{\mu}}\left(\xi_{\mu}\right)$, such that 
- for every $(x, \xi) \in Q_{\mu}^{1}$ one has

$$
2^{-4}(R+|\xi|) \leq M_{\mu} \leq 2^{-3}(R+|\xi|)
$$

- $\stackrel{\circ}{Q_{\mu}^{1} \cap} \stackrel{\circ}{Q_{\mu^{\prime}}^{1}}=\emptyset$ for each $\mu \neq \mu^{\prime}\left(\stackrel{\circ}{Q_{\mu}^{1}}\right.$ is the interior of $\left.Q_{\mu}^{1}\right)$ and $\bigcup_{\mu \in \mathbb{N}} Q_{\mu}^{1}=\mathbb{R}^{4}$.

Remark 2.3 For every $(x, \xi) \in 2 Q_{\mu}^{1}$ one has ${ }^{1}$

$$
17^{-1}(R+|\xi|) \leq M_{\mu} \leq 7^{-1}(R+|\xi|) .
$$

Denoting by $\left(x_{\mu}, \xi_{\mu}\right)$ the center of $Q_{\mu}^{1}$ one has in fact

$$
(R+|\xi|) \leq\left(R+\left|\xi_{\mu}\right|\right)+\left|\xi-\xi_{\mu}\right| \leq\left(1+2^{4}\right) M_{\mu}
$$

and

$$
M_{\mu} \leq 2^{-3}(R+|\xi|)+2^{-3}\left|\xi-\xi_{\mu}\right| \leq 2^{-3}(R+|\xi|)+2^{-3} M_{\mu} .
$$

As a consequence, one gets $M_{\mu^{\prime}} \sim M_{\mu}$ whenever $2 Q_{\mu}^{1} \cap 2 Q_{\mu^{\prime}}^{1} \neq \emptyset$.

Remark 2.3 allows us to apply Proposition A.2, so that the metric

$$
g_{(x, \xi)}^{I}(y, \eta)=|y|^{2}+\frac{|\eta|^{2}}{M_{\mu}^{2}}, \quad(x, \xi) \in \stackrel{\circ}{Q}_{\mu},
$$

can be extended to a Hörmander metric $g^{I}$. As a consequence of Theorem A.5 and of Lemma 2.2 we can finally reduce the proof of Theorem 1.2 to the proof of the following theorem which can be considered as its microlocal version.

Theorem 2.4 With the same hypotheses of Theorem 1.2, let $\left\{\psi_{\mu}\right\}_{\mu \in \mathbb{N}}$, $\left\{\varphi_{\mu}\right\}_{\mu \in \mathbb{N}}$ be the partition of unity associated with the covering $\left\{Q_{\mu}^{1}\right\}_{\mu \in \mathbb{N}}$ in the sense of Proposition A.4. Then ${ }^{2}$

$$
\left\|\left(p \psi_{\mu}\right)^{w} \varphi_{\mu}^{w} u\right\|_{0}^{2} \gtrsim\left\|\left(q \psi_{\mu}\right)^{w} \varphi_{\mu}^{w} u\right\|_{0}^{2}-\left(r_{\mu}^{w} u, u\right)_{L^{2}}, \quad \forall \mu \in \mathbb{N}, \forall u \in \mathcal{S}\left(\mathbb{R}^{2}\right),
$$

with $p$ as in Definition 2.1 and $\sum_{\mu \in \mathbb{N}} r_{\mu}^{w} \in \mathcal{L}\left(L^{2}\left(\mathbb{R}^{2}\right), L^{2}\left(\mathbb{R}^{2}\right)\right)$.

\footnotetext{
${ }^{1}$ In what follows, given a rectangle $Q$ in $\mathbb{R}^{4}$ and $k \in \mathbb{R}_{+}$, we denote by $k Q$ the dilated of $Q$ by the factor $k$ keeping its center fixed

${ }^{2}$ From now on, for two non negative functions $f, g$ on $\mathbb{R}^{n}$ we write $f \lesssim g$ (resp., $f \gtrsim g$ ) when there exists a constant $c>0$ such that $f(x, \xi) \leq c g(x, \xi)$ (resp., $f(x, \xi) \geq c g(x, \xi)$ ). We simply write $f \sim g$ when $f \lesssim g$ and $f \gtrsim g$. For function families $\left\{f_{k}\right\}_{k},\left\{g_{k}\right\}_{k}$ and $\left\{h_{k}\right\}_{k}$ we write $f_{k} \lesssim g_{k}$ (resp., $f_{k} \gtrsim g_{k}$ ) whenever the constants in the inequalities don't depend on $k ; f_{k} \gtrsim g_{k}-h_{k}$ whenever $f_{k}+h_{k} \gtrsim g_{k}$.
} 
The proof of Theorem 2.4 requires a second microlocalization. Let $\left\{Q_{\mu}^{1}\right\}_{\mu \in \mathbb{N}}$ be the partition of $\mathbb{R}^{4}$ just described, we refer to the $Q_{\mu}^{1}$ as "rectangles of first generation". Among them, we retain those that satisfy one of the following conditions:

either there exists $(x, \xi) \in Q_{\mu}^{1}$ such that $1 \leq 2[R /(R+|\xi|)]^{1 /(h+1)}$

or $p(x, \xi)>0$ for every $(x, \xi) \in 4 Q_{\mu}^{1}$.

If both these conditions fail we divide $Q_{\mu}^{1}$ into $2^{2(h+1)}$ subrectangles $Q_{\mu^{\prime}}^{2}$ (rectangles of second generation) by partitioning $I_{1}\left(x_{\mu}\right)$ into four congruent rectangles and $I_{M_{\mu}}\left(\xi_{\mu}\right)$ into $4^{h}$ congruent rectangles. We denote by $\operatorname{diam}_{x} Q_{\mu^{\prime}}^{2}, \operatorname{diam}_{\xi} Q_{\mu^{\prime}}^{2}$ respectively their $x$-diameter and $\xi$-diameter, and retain those satisfying one of the following conditions, for $j=2$,

$$
\begin{gathered}
\exists(x, \xi) \in Q_{\mu^{\prime}}^{j} \text { such that } \operatorname{diam}_{x} Q_{\mu^{\prime}}^{j} \leq \frac{2 R^{1 /(h+1)}}{(R+|\xi|)^{1 /(h+1)}} \\
p(x, \xi)>0, \quad \forall(x, \xi) \in 4 Q_{\mu^{\prime}}^{j}
\end{gathered}
$$

We again divide the rectangles that were not retained into $2^{2(h+1)}$ subrectangles, $Q_{\mu^{\prime}}^{3}$, as described above and retain those satisfying one of conditions $(2.5),(2.6)$ for $j=3$. We continue this process and after a finite number of steps (because of the condition (2.5)) we obtain a partition of $\mathbb{R}^{4}$.

Lemma 2.5 Let $Q_{\mu}^{j}$ be a rectangle, either retained or not, of the $j$-th generation, we then have

$$
2^{-4}(R+|\xi|)\left(\operatorname{diam}_{x} Q_{\mu}^{j}\right)^{h} \leq \operatorname{diam}_{\xi} Q_{\mu}^{j} \leq 2^{-3}(R+|\xi|)\left(\operatorname{diam}_{x} Q_{\mu}^{j}\right)^{h},
$$

$\forall(x, \xi) \in Q_{\mu}^{j}$, and

$$
2^{-1}(R+|\xi|) \leq R+|\eta| \leq 2(R+|\xi|), \quad(x, \xi), \quad(y, \eta) \in 4 Q_{\mu}^{j} .
$$

Proof. When $j=1,(2.7)$ is trivial. When $j \geq 2$, since

$$
\operatorname{diam}_{x} Q_{\mu}^{j}=(1 / 2) \operatorname{diam}_{x} Q_{\mu}^{j-1} \text { and } \operatorname{diam}_{\xi} Q_{\mu}^{j}=\left(1 / 2^{h}\right) \operatorname{diam}_{\xi} Q_{\mu}^{j-1},
$$

(2.7) follows by induction.

Consider now $(x, \xi) \in 4 Q_{\mu}^{j}, j \geq 1$. From (2.7), taking into account that $\operatorname{diam}_{x} Q_{\mu}^{j} \leq 1$, it follows, on denoting by $(\bar{x}, \bar{\xi})$ the center of $Q_{\mu}^{j}$, that $|\xi-\bar{\xi}| \leq 2 \operatorname{diam}_{\xi} Q_{\mu}^{j} \leq 2^{-2}(R+|\bar{\xi}|)$. Thus

$$
R+|\xi| \leq R+|\bar{\xi}|+|\xi-\bar{\xi}| \leq 5 / 4(R+|\bar{\xi}|) \quad \text { and } \quad R+|\xi| \geq 3 / 4(R+|\bar{\xi}|) .
$$

This concludes the proof. 
Definition 2.6 Let $\left\{Q_{\mu \nu}\right\}(\mu \in \mathbb{N}, \nu=1, \ldots, N(\mu))$ be the partition of $\mathbb{R}^{4}$ obtained by means of conditions (2.5) and (2.6). The first index, $\mu$, indicates the rectangle of the first generation $Q_{\mu}^{1}$ whose decomposition gives $Q_{\mu \nu}$. We denote by $\left(x_{\mu \nu}, \xi_{\mu \nu}\right)$ the center of $Q_{\mu \nu}$, by $\delta_{\mu \nu}$ and $\epsilon_{\mu \nu}$ respectively its $x$ diameter and the $\xi$-diameter and, finally, we write $h_{\mu \nu}=\left(\delta_{\mu \nu} \epsilon_{\mu \nu}\right)^{-1}$.

The following propositions describe the basic properties of the partition.

Proposition 2.7 If $(x, \xi) \in Q_{\mu \nu}$, then

$$
\begin{gathered}
2^{-4}(R+|\xi|) \delta_{\mu \nu}^{h} \leq \epsilon_{\mu \nu} \leq 2^{-3}(R+|\xi|) \delta_{\mu \nu}^{h} ; \\
\frac{R^{1 /(h+1)}}{(R+|\xi|)^{1 /(h+1)}} \leq \delta_{\mu \nu} \leq 1 ; \\
2^{3}(R+|\xi|)^{-1} \delta_{\mu \nu}^{-h-1} \leq h_{\mu \nu} \leq 2^{4}(R+|\xi|)^{-1} \delta_{\mu \nu}^{-h-1}<16 / R .
\end{gathered}
$$

Moreover, if $Q_{\mu \nu}$ is retained by virtue of (2.5) one has

$$
\begin{gathered}
2^{2-h} R^{-1} \leq h_{\mu \nu} ; \\
\delta_{\mu \nu} \leq 2^{(h+2) /(h+1)} \frac{R^{1 /(h+1)}}{(R+|\xi|)^{1 /(h+1)}}, \quad \forall(x, \xi) \in 4 Q_{\mu \nu} .
\end{gathered}
$$

Proof. Inequality (2.9) is simply (2.7) written in this case. Inequality (2.10) is trivial if $\delta_{\mu \nu}=1$. If $Q_{\mu \nu}$ is obtained by dividing some $Q_{\mu}^{j}$ this means, in particular, that $Q_{\mu}^{j}$ does not satisfy (2.5). Hence, taking into account that $2 \delta_{\mu \nu}=\operatorname{diam}_{x} Q_{\mu}^{j}$ one proves (2.10) also in this case. Inequality (2.11) is obtained by multiplying (2.9) by $\delta_{\mu \nu}$ and by using (2.10).

Let us suppose now that $Q_{\mu \nu}$ is retained because of (2.5). From (2.11) we get (2.12). Finally, (2.13) is deduced from (2.5) and (2.8). sition.

Same arguments used in Proposition 1.4 of [7] prove the following Propo-

Proposition 2.8 If $2 Q_{\mu \nu} \cap 2 Q_{\mu^{\prime} \nu^{\prime}} \neq \emptyset$ then

$$
2^{-6} \delta_{\mu^{\prime} \nu^{\prime}} \leq \delta_{\mu \nu} \leq 2^{6} \delta_{\mu^{\prime} \nu^{\prime}} \quad 2^{-(6 h+3)} \epsilon_{\mu^{\prime} \nu^{\prime}} \leq \epsilon_{\mu \nu} \leq 2^{6 h+3} \epsilon_{\mu^{\prime} \nu^{\prime}} .
$$

In view of the results above we can apply Proposition A.2 and obtain that the metric

$$
g_{(x, \xi)}^{I I}(y, \eta)=\delta_{\mu \nu}^{-2}|y|^{2}+\epsilon_{\mu \nu}^{-2}|\eta|^{2}, \quad(x, \xi) \in \stackrel{\circ}{Q}_{\mu \nu}
$$

can be extended to a Hörmander metric $g^{I I}$ with Planck function $h_{I I}$ given by

$$
h_{I I}(x, \xi)=h_{\mu \nu}, \quad(x, \xi) \in \stackrel{\circ}{\mu \nu}
$$




\section{Remark 2.9}

1) If $Q_{\mu \nu}$ is obtained by partitioning a cube $Q_{\mu}^{j}$ of generation $j \geq 1$, then one has $4 Q_{\mu}^{j} \cap \Sigma \neq \emptyset$ and it easily follows

$$
\left|\xi_{1}\right| \leq 2^{2+h} \epsilon_{\mu \nu}, \quad\left|x_{1}\right| \leq 2^{3} \delta_{\mu \nu}, \quad \forall\left(x_{1}, x_{2}, \xi_{1}, \xi_{2}\right) \in 2 Q_{\mu \nu}
$$

2) If $Q_{\mu \nu}$ is retained by means of (2.6) then

$$
\text { either }\left|x_{1}\right|>\frac{\delta_{\mu \nu}}{\sqrt{2}} \quad \text { or } \quad\left|\xi_{1}\right|>\frac{\epsilon_{\mu \nu}}{\sqrt{2}}, \quad \forall\left(x_{1}, x_{2}, \xi_{1}, \xi_{2}\right) \in 2 Q_{\mu \nu} .
$$

In fact, if by contradiction we suppose that there exists $\left(\bar{x}_{1}, \bar{x}_{2}, \bar{\xi}_{1}, \bar{\xi}_{2}\right) \in 2 Q_{\mu \nu}$ such that $\left|\bar{x}_{1}\right| \leq \delta_{\mu \nu} / \sqrt{2}$ and $\left|\bar{\xi}_{1}\right| \leq \epsilon_{\mu \nu} / \sqrt{2}$, this implies $\left(0, \bar{x}_{2}, 0, \bar{\xi}_{2}\right) \in$ $4 Q_{\mu \nu} \cap \Sigma$. In particular, if $Q_{\mu \nu}$ is retained by means of $(2.6)$ then $p(x, \xi)+$ $R^{2} \gtrsim \epsilon_{\mu \nu}^{2}$ for every $(x, \xi) \in 2 Q_{\mu \nu}$.

It is also convenient to compare the metric $g^{I I}$ with the metric $G$ of Definition 1.1 .

Lemma 2.10 For every $\mu \in \mathbb{N}$ and $\nu=1, \ldots, N(\mu)$ the following relations hold

$$
D_{\Sigma}(x, \xi) \sim \delta_{\mu \nu}^{h}, \quad\left(R^{2}+|\xi|^{2}\right)^{1 / 2} D_{\Sigma}(x, \xi) \sim \epsilon_{\mu \nu}, \quad \forall(x, \xi) \in Q_{\mu \nu} .
$$

Proof. By (2.9) it is enough to prove only one of the relations.

If $Q_{\mu \nu}$ is obtained by partition from a rectangle $Q_{\mu}^{j}$ of generation $j \geq 1$ then, by Remark 2.9, (2.9) and (2.10), $D_{\Sigma}(x, \xi) \lesssim \delta_{\mu \nu}^{h}$ on that rectangle. If it is a first generation rectangle the same holds because of $\delta_{\mu \nu}=1$ and $D_{\Sigma}(x, \xi) \lesssim 1$.

The inequality $\delta_{\mu \nu}^{h} \lesssim D_{\Sigma}(x, \xi)$ follows from (2.13) when $Q_{\mu \nu}$ is retained by means of (2.5) and by Remark 2.9 and (2.9) in the other case.

As an immediate consequence of Lemma 2.10 and of Remark A.1 we get that $G$ is equivalent to $g^{I I}$. Moreover, since the radius $r$ of the balls $B_{\left(x_{j}, \xi_{j}\right)}^{G}(r)=\left\{(y, \eta) \in \mathbb{R}^{4}: G_{\left(x_{j}, \xi_{j}\right)}\left(y-x_{j}, \eta-\xi_{j}\right) \leq r^{2}\right\}$ satisfies the condition $r \leq c_{S V}$ (see (1.5)), Lemma 2.10 allows us to compare these balls with the rectangles $Q_{\mu \nu}$.

\section{Corollary 2.11}

1) The metric $G$ of Definition 1.1 is equivalent to the metric $g^{I I}$.

2) There exists a positive constant $k$, independent of $\mu, \nu$, such that

$$
Q_{\mu \nu} \subseteq \bigcup_{j \in \mathcal{J}_{\mu \nu}} B_{\left(x_{j}, \xi_{j}\right)}^{G}(r) \subseteq k Q_{\mu \nu}, \quad \forall \mu \in \mathbb{N}, \nu=1, \ldots, N(\mu),
$$

where $\mathcal{J}_{\mu \nu}=\left\{j \in \mathbb{N}: B_{\left(x_{j}, \xi_{j}\right)}^{G}(r) \cap Q_{\mu \nu} \neq \emptyset\right\}$. 
Definition 2.12 For every $\mu \in \mathbb{N}$ we define

$$
\rho_{\mu}=\left(\frac{R}{2^{4} M_{\mu}}\right)^{1 /(h+1)} .
$$

Note that from (2.2) and (2.10) one has

$$
\rho_{\mu} \leq \frac{R^{1 /(h+1)}}{(R+|\xi|)^{1 /(h+1)}} \leq \delta_{\mu \nu}, \quad(x, \xi) \in Q_{\mu \nu}, \mu \in \mathbb{N}, \nu=1, \ldots, N(\mu)
$$

Proposition 2.13 Let $p \in S^{2}\left(\mathbb{R}^{2}\right)$ be the symbol defined in Definition 2.1 and $\left\{\psi_{\mu}\right\}_{\mu},\left\{\varphi_{\mu}\right\}_{\mu}$ be the partition of unity associated to the covering $\left\{Q_{\mu}^{1}\right\}$ in the sense of Proposition A.4 then $\rho_{\mu}^{2} p \psi_{\mu} \in S\left(h_{I I}^{-2}, g^{I I}\right)$ uniformly in $\mu$.

Proof. Let us recall that, by construction, $\operatorname{supp} \psi_{\mu} \subseteq 2 Q_{\mu}^{1}$ and

$$
\left|\partial_{x}^{\alpha} \partial_{\xi}^{\beta} \psi_{\mu}(x, \xi)\right| \lesssim M_{\mu}^{-|\beta|}, \quad \forall \alpha, \beta \in \mathbb{Z}_{+}^{2} .
$$

We have to evaluate the seminorms of $\rho_{\mu}^{2} p \psi_{\mu}$ only on rectangles $Q_{\mu^{\prime} \nu^{\prime}}$ such that $Q_{\mu^{\prime} \nu^{\prime}} \cap 2 Q_{\mu}^{1} \neq \emptyset$. Hence, by Remark 2.3, $M_{\mu} \sim M_{\mu^{\prime}}$ and $\rho_{\mu} \sim \rho_{\mu^{\prime}}$. Thus

$$
\left|\partial_{x}^{\alpha} \partial_{\xi}^{\beta} \psi_{\mu}(x, \xi)\right| \lesssim M_{\mu^{\prime}}^{-|\beta|} \lesssim \epsilon_{\mu^{\prime} \nu^{\prime}}^{-|\beta|} \delta_{\mu^{\prime} \nu^{\prime}}^{-|\alpha|+(h|\beta|+|\alpha|)} \lesssim \epsilon_{\mu^{\prime} \nu^{\prime}}^{-|\beta|} \delta_{\mu^{\prime} \nu^{\prime}}^{-|\alpha|}, \quad(x, \xi) \in Q_{\mu^{\prime}, \nu^{\prime}}
$$

We now have to show that

$$
\left|\partial_{x}^{\alpha} \partial_{\xi}^{\beta}\left(\rho_{\mu}^{2} p\right)(x, \xi)\right| \lesssim \epsilon_{\mu^{\prime} \nu^{\prime}}^{2-|\beta|} \delta_{\mu^{\prime} \nu^{\prime}}^{2-|\alpha|}, \quad(x, \xi) \in Q_{\mu^{\prime} \nu^{\prime}}
$$

Note that, for $(x, \xi) \in Q_{\mu^{\prime} \nu^{\prime}}$ and $|\alpha|+|\beta| \geq 2 h$,

$$
\left|\partial_{x}^{\alpha} \partial_{\xi}^{\beta}\left(\rho_{\mu}^{2} p\right)(x, \xi)\right| \lesssim \rho_{\mu^{\prime}}^{2} M_{\mu^{\prime}}^{2-|\beta|} \lesssim\left(\epsilon_{\mu^{\prime} \nu^{\prime}} \delta_{\mu^{\prime} \nu^{\prime}}^{-h}\right)^{2-|\beta|} \delta_{\mu^{\prime} \nu^{\prime}}^{2} \lesssim \epsilon_{\mu^{\prime} \nu^{\prime}}^{2-|\beta|} \delta_{\mu^{\prime} \nu^{\prime}}^{2-|\alpha|}
$$

If $Q_{\mu^{\prime} \nu^{\prime}}$ is a retained rectangle of first generation, it must be $\delta_{\mu^{\prime} \nu^{\prime}}=1$ and $\epsilon_{\mu^{\prime} \nu^{\prime}}=M_{\mu^{\prime}}$, so that on $Q_{\mu^{\prime} \nu^{\prime}}$ one gets

$$
\rho_{\mu}^{2} p(x, \xi) \lesssim \rho_{\mu}^{2} M_{\mu^{\prime}}^{2} \lesssim \delta_{\mu^{\prime} \nu^{\prime}}^{2} \epsilon_{\mu^{\prime} \nu^{\prime}}^{2}
$$

If $Q_{\mu^{\prime} \nu^{\prime}}$ is obtained by partition from $Q_{\mu^{\prime}}^{j}$, Remark 2.9 and (2.9) give, for every $(x, \xi) \in 2 Q_{\mu^{\prime} \nu^{\prime}}$

$$
|p(x, \xi)| \lesssim \epsilon_{\mu^{\prime} \nu^{\prime}}^{2}+\delta_{\mu^{\prime} \nu^{\prime}}^{2 h}(R+|\xi|)^{2} \lesssim \epsilon_{\mu^{\prime} \nu^{\prime}}^{2}
$$

and this means that estimate (2.18) holds with $|\alpha|+|\beta|=0$. The remaining cases in (2.18) are recovered by interpolation. Finally, by using the Leibniz Formula we complete the proof. 
Proposition 2.14 If $q \in S^{2}\left(\mathbb{R}^{2}\right)$ satisfies hypotheses of Theorem 2.4 then, with the same notation of Proposition 2.13, $\rho_{\mu}^{2} q \psi_{\mu} \in S\left(h_{I I}^{-2}, g^{I I}\right)$ uniformly in $\mu$.

Proof. Since $q \in S^{2}\left(\mathbb{R}^{2}\right)$, the same arguments used in the proof of Proposition 2.13 show that, when $|\alpha|+|\beta|$ is large enough,

$$
\left|\partial_{x}^{\alpha} \partial_{\xi}^{\beta}\left(\rho_{\mu}^{2} q \psi_{\mu}\right)(x, \xi)\right| \lesssim \epsilon_{\mu^{\prime} \nu^{\prime}}^{2-|\beta|} \delta_{\mu^{\prime} \nu^{\prime}}^{2-|\alpha|}, \quad(x, \xi) \in Q_{\mu^{\prime} \nu^{\prime}}
$$

Thus, it is enough to prove the estimate for $|\alpha|+|\beta|=0$ to complete the proof as we did before. If $Q_{\mu^{\prime} \nu^{\prime}}$ is a retained rectangle of first generation, we have $\delta_{\mu^{\prime} \nu^{\prime}}=1$ and $\epsilon_{\mu^{\prime} \nu^{\prime}}=M_{\mu^{\prime}}$ so that, on $Q_{\mu^{\prime} \nu^{\prime}}$,

$$
\rho_{\mu}^{2}\left|\left(q \psi_{\mu}\right)(x, \xi)\right| \lesssim \rho_{\mu^{\prime}}^{2} M_{\mu^{\prime}}^{2} \lesssim \delta_{\mu^{\prime} \nu^{\prime}}^{2} \epsilon_{\mu^{\prime} \nu^{\prime}}^{2}
$$

Assume now that $Q_{\mu^{\prime} \nu^{\prime}}$ is obtained by partitioning some $Q_{\mu^{\prime}}^{j}$ and denote by $B_{j}$ the ball

$$
B_{\left(x_{j}, \xi_{j}\right)}^{G}(r)=\left\{(y, \eta) \in \mathbb{R}^{4}: G_{\left(x_{j}, \xi_{j}\right)}\left(y-x_{j}, \eta-\xi_{j}\right) \leq r^{2}\right\}
$$

with $j \in \mathbb{N}$. By Corollary 2.11 and by (1.6) we get

$$
\max _{Q_{\mu^{\prime} \nu^{\prime}}}|q| \leq \sup _{j \in \mathcal{J}_{\mu^{\prime} \nu^{\prime}}}\left(\max _{B_{j}}|q|\right) \lesssim \sup _{j \in \mathcal{J}_{\mu^{\prime} \nu^{\prime}}}\left(\max _{B_{j}} L\right) \lesssim \max _{k Q_{\mu^{\prime} \nu^{\prime}}} L
$$

with $\mathcal{J}_{\mu^{\prime} \nu^{\prime}}=\left\{j \in \mathbb{N}: B_{j} \cap Q_{\mu^{\prime} \nu^{\prime}} \neq \emptyset\right\}$. Therefore the proof is complete if we show that

$$
\max _{k Q_{\mu^{\prime} \nu^{\prime}}} L \lesssim \epsilon_{\mu^{\prime} \nu^{\prime}}^{2}
$$

To this purpose, denote by $\left(\bar{x}_{1}, \bar{x}_{2}, \bar{\xi}_{1}, \bar{\xi}_{2}\right) \in \mathbb{R}^{4}$ the center of $k Q_{\mu^{\prime} \nu^{\prime}}$. From 1$)$ of Remark 2.9 it follows, for every $\left(x_{1}, x_{2}, \xi_{1}, \xi_{2}\right) \in k Q_{\mu^{\prime} \nu^{\prime}}$,

$\left|x_{1}\right| \leq\left|\bar{x}_{1}\right|+\left|x_{1}-\bar{x}_{1}\right| \leq\left(2^{3}+k\right) \delta_{\mu^{\prime} \nu^{\prime}}$ and $\left|\xi_{1}\right| \leq\left|\bar{\xi}_{1}\right|+\left|\xi_{1}-\bar{\xi}_{1}\right| \leq\left(2^{2+h}+k\right) \epsilon_{\mu^{\prime} \nu^{\prime}}$.

Moreover, by virtue of (2.9),

$$
\begin{aligned}
\left|\xi_{2}\right|^{2} & \leq\left(R^{2}+|\xi|^{2}\right) \leq 2|\xi-\bar{\xi}|^{2}+2\left(R^{2}+|\bar{\xi}|^{2}\right) \\
& \leq 2 k^{2} \epsilon_{\mu^{\prime} \nu^{\prime}}^{2}+2^{9} \frac{\epsilon_{\mu^{\prime} \nu^{\prime}}^{2}}{\delta_{\mu^{\prime} \nu^{\prime}}^{2 h}} \leq\left(2 k^{2}+2^{9}\right) \frac{\epsilon_{\mu^{\prime} \nu^{\prime}}^{2}}{\delta_{\mu^{\prime} \nu^{\prime}}^{2 h}}
\end{aligned}
$$

whence, for every $(x, \xi) \in k Q_{\mu^{\prime} \nu^{\prime}}$, one gets

$$
L(x, \xi)=\xi_{1}^{2}+x_{1}^{2 h} \xi_{2}^{2} \lesssim \epsilon_{\mu^{\prime} \nu^{\prime}}^{2}
$$




\section{Proof of Theorem 2.4}

The proof of Theorem 2.4 requires some preliminary results whose aim is to microlocalize estimate (2.4) on the rectangles $Q_{\mu \nu}$ we constructed. The first step is a subelliptic estimate for $p^{w}$ that we exploit in the sequel.

Theorem 3.1 Let $p \in S^{2}\left(\mathbb{R}^{2}\right)$ be the symbol introduced in Definition 2.1 and let $\left\{\varphi_{\mu}\right\}_{\mu \in \mathbb{N}},\left\{\psi_{\mu}\right\}_{\mu \in \mathbb{N}}$ the partition of unity associated to the covering $\left\{Q_{\mu}^{1}\right\}$ in the sense of Proposition A.4. Let us consider a bounded family $\left\{\phi_{\mu}\right\}_{\mu \in \mathbb{N}}$ in $S\left(1, g^{I}\right)$ with $\phi_{\mu}=1$ on $(9 / 8) Q_{\mu}^{1}$ and $\operatorname{supp} \phi_{\mu} \subseteq(5 / 4) Q_{\mu}^{1}$. Then the following estimates hold

$$
\begin{aligned}
\left\|\left(p \psi_{\mu}\right)^{w} \varphi_{\mu}^{w} u\right\|_{0}^{2} \gtrsim & M_{\mu}^{4 /(h+1)}\left\|\varphi_{\mu}^{w} u\right\|_{0}^{2}-\left\|\left(1-\psi_{\mu}\right)^{w}\left(M_{\mu}^{2} \varphi_{\mu}^{w}\right) u\right\|_{0}^{2} \\
& \quad-\left\|\left(1-\phi_{\mu}\right)^{w}\left(M_{\mu}^{2} \varphi_{\mu}^{w}\right) u\right\|_{0}^{2}-\left\|\varphi_{\mu}^{w} u\right\|_{0}^{2}, \quad u \in \mathcal{S}\left(\mathbb{R}^{2}\right) .
\end{aligned}
$$

Proof. Let us denote by $U_{1}$ the set of $\mu \in \mathbb{N}$ such that the center

$$
\left(x_{1, \mu}, x_{2, \mu}, \xi_{1, \mu}, \xi_{2, \mu}\right)=\left(x_{\mu}, \xi_{\mu}\right)
$$

of $Q_{\mu}^{1}$ satisfies the following condition: either $\left|x_{1, \mu}\right| \geq 2$ or $\left|\xi_{1, \mu}\right| \geq 2\left|\xi_{2, \mu}\right|$. By $U_{2}$ we denote the set $\mathbb{N} \backslash U_{1}$. The following relations hold for every $(x, \xi) \in 2 Q_{\mu}^{1}$

$$
\exists \gamma_{1}>0 \text { such that } p(x, \xi)+R^{2} \geq \gamma_{1} M_{\mu}^{2}, \quad \forall \mu \in U_{1} ;
$$

$$
\exists \gamma_{2}>0 \text { such that } p(x, \xi)+R^{2} \geq \gamma_{2}\left(\xi_{1}^{2}+x_{1}^{2 h} M_{\mu}^{2}\right), \quad \forall \mu \in U_{2} .
$$

The ellipticity property (3.2) gives

$$
\left\|\left(p \psi_{\mu}\right)^{w} \varphi_{\mu}^{w} u\right\|_{0}^{2} \gtrsim M_{\mu}^{4}\left\|\varphi_{\mu}^{w} u\right\|_{0}^{2}-\left\|\left(1-\psi_{\mu}\right)^{w} M_{\mu}^{2} \varphi_{\mu}^{w} u\right\|_{0}^{2}-\left\|\varphi_{\mu}^{w} u\right\|_{0}^{2},
$$

$u \in \mathcal{S}\left(\mathbb{R}^{2}\right), \forall \mu \in U_{1}$. We now prove

$$
\left\|\left(p \psi_{\mu}\right)^{w} \varphi_{\mu}^{w} u\right\|_{0}^{2} \gtrsim M_{\mu}^{4 /(h+1)}\left\|\varphi_{\mu}^{w} u\right\|_{0}^{2}-\left\|\left(1-\phi_{\mu}\right)^{w} M_{\mu}^{2} \varphi_{\mu}^{w} u\right\|_{0}^{2}-\left\|\varphi_{\mu}^{w} u\right\|_{0}^{2},
$$

$u \in \mathcal{S}\left(\mathbb{R}^{2}\right), \forall \mu \in U_{2}$. Note that in view of (3.3), for $\mu \in U_{2}$ one has

$$
p \psi_{\mu}+R^{2}=\left(p+R^{2}\right) \psi_{\mu}+\left(1-\psi_{\mu}\right) R^{2} \geq \gamma_{2}\left(\xi_{1}^{2}+x_{1}^{2 h} M_{\mu}^{2}\right) \psi_{\mu},
$$

and that $p \psi_{\mu}+R^{2}-\gamma_{2}\left(\xi_{1}^{2}+x_{1}^{2 h} M_{\mu}^{2}\right) \psi_{\mu} \in S\left(h_{g^{I}}^{-2}, g^{I}\right)$. This allows an application of the Fefferman-Phong inequality (see [10, Lemma 18.6.10]). Taking into account that the lowest eigenvalue of the anharmonic oscillator $D_{x_{1}}^{2}+M_{\mu}^{2} x_{1}^{2 h}$ is $\lambda \sim M_{\mu}^{2 /(h+1)}$, we get for every $u \in \mathcal{S}\left(\mathbb{R}^{2}\right)$

$$
\begin{aligned}
\left\|\left(p \psi_{\mu}\right)^{w} \phi_{\mu}^{w} u\right\|_{0}^{2} \gtrsim & M_{\mu}^{4 /(h+1)}\left\|\phi_{\mu}^{w} u\right\|_{0}^{2} \\
& -\left\|\left(\left(\xi_{1}^{2}+M_{\mu}^{2} x_{1}^{2 h}\right)\left(1-\psi_{\mu}\right)\right)^{w} \phi_{\mu}^{w} u\right\|_{0}^{2}-\left\|\phi_{\mu}^{w} u\right\|_{0}^{2} .
\end{aligned}
$$


In order to estimate the term $\left\|\left(\left(\xi_{1}^{2}+M_{\mu}^{2} x_{1}^{2 h}\right)\left(1-\psi_{\mu}\right)\right)^{w} \phi_{\mu}^{w} u\right\|_{0}^{2}$, let us now consider the Hörmander metric $G_{\mu}$ defined by

$$
G_{\mu,(x, \xi)}(y, \eta)=\frac{|y|^{2}}{\left\langle x-x_{\mu}\right\rangle^{2}}+\frac{|\eta|^{2}}{M_{\mu}^{2}+\left|\xi-\xi_{\mu}\right|^{2}}, \quad(x, \xi) \in \mathbb{R}^{4} .
$$

Note that $G_{\mu} \sim g^{I}$ on $2 Q_{\mu}^{1}$. Moreover $\xi_{1}^{2}+x_{1}^{2 h} M_{\mu}^{2} \in S\left(m, G_{\mu}\right)$, with (admissible) weight $m(x, \xi)=\left\langle x-x_{\mu}\right\rangle^{2 h}\left(M_{\mu}^{2}+\left|\xi-\xi_{\mu}\right|^{2}\right)$, and $1-\psi_{\mu}, \phi_{\mu} \in S\left(1, G_{\mu}\right)$ with seminorms independent of $\mu$. Thus $\left(\xi_{1}^{2}+M_{\mu}^{2} x_{1}^{2 h}\right)\left(1-\psi_{\mu}\right) \sharp \phi_{\mu} \in S\left(1, G_{\mu}\right)$ (if $a \in S\left(m_{1}, g\right)$ and $b \in S\left(m_{2}, g\right), a \sharp b \in S\left(m_{1} m_{2}, g\right)$ denotes the symbol of the composition $a^{w} b^{w}$ ) with seminorms not depending on $\mu$. Hence, from (3.6), we get

$$
\begin{aligned}
\left\|\left(p \psi_{\mu}\right)^{w} \phi_{\mu}^{w} \varphi_{\mu}^{w} u\right\|_{0}^{2} & \gtrsim\left\|\phi_{\mu}^{w} \varphi_{\mu}^{w} M_{\mu}^{2 /(h+1)} u\right\|_{0}^{2}-\left\|\varphi_{\mu}^{w} u\right\|_{0}^{2} \\
& \gtrsim M_{\mu}^{4 /(h+1)}\left\|\varphi_{\mu}^{w} u\right\|_{0}^{2}-M_{\mu}^{4 /(h+1)}\left\|\left(1-\phi_{\mu}\right)^{w} \varphi_{\mu}^{w} u\right\|_{0}^{2}-\left\|\varphi_{\mu}^{w} u\right\|_{0}^{2},
\end{aligned}
$$

for $u \in \mathcal{S}\left(\mathbb{R}^{2}\right)$. From this the conclusion follows once we observe that

$$
\left\|\left(p \psi_{\mu}\right)^{w} \phi_{\mu}^{w} \varphi_{\mu}^{w} u\right\|_{0}^{2} \lesssim\left\|\left(p \psi_{\mu}\right)^{w} \varphi_{\mu}^{w} u\right\|_{0}^{2}+\left\|\left(\frac{p \psi_{\mu}}{M_{\mu}^{2}}\right)^{w}\left(1-\phi_{\mu}\right)^{w} M_{\mu}^{2} \varphi_{\mu}^{w} u\right\|_{0}^{2}, u \in \mathcal{S}\left(\mathbb{R}^{2}\right)
$$

with $p \psi_{\mu} / M_{\mu}^{2} \in S\left(1, g^{I I}\right)$ uniformly in $\mu$. The proof of the theorem readily follows from (3.4) and (3.5).

Theorem 3.1 is used to prove the following Proposition.

Proposition 3.2 Let $p$ and $q$ be as in Theorem 2.4. Then estimate (2.4) holds provided one has

$$
\left\|\left(\rho_{\mu}^{2} p \psi_{\mu}\right)^{w} u\right\|_{0}^{2} \gtrsim\left\|\left(\rho_{\mu}^{2} q \psi_{\mu}\right)^{w} u\right\|_{0}^{2}-\|u\|_{0}^{2}-\left(r_{\mu, 1}^{w} u, u\right)_{L^{2}},
$$

$\forall u \in \mathcal{S}\left(\mathbb{R}^{2}\right), \forall \mu \in \mathbb{N}$, where $\sum_{\mu \in \mathbb{N}} \rho_{\mu}^{-4} \varphi_{\mu}^{w} r_{\mu, 1}^{w} \varphi_{\mu}^{w} \in \mathcal{L}\left(L^{2}\left(\mathbb{R}^{2}\right), L^{2}\left(\mathbb{R}^{2}\right)\right)$.

Proof. We divide all terms in (3.7) by $\rho_{\mu}^{4}$ and write $\varphi_{\mu}^{w} u$ instead of $u$. From (2.16) we then have

$$
\left\|\left(p \psi_{\mu}\right)^{w} \varphi_{\mu}^{w} u\right\|_{0}^{2} \gtrsim\left\|\left(q \psi_{\mu}\right)^{w} \varphi_{\mu}^{w} u\right\|_{0}^{2}-M_{\mu}^{4 /(h+1)}\left\|\varphi_{\mu}^{w} u\right\|_{0}^{2}-\left(\rho_{\mu}^{-4} \varphi_{\mu}^{w} r_{\mu, 1}^{w} \varphi_{\mu}^{w} u, u\right)_{L^{2}},
$$

$\forall u \in \mathcal{S}\left(\mathbb{R}^{2}\right)$. The conclusion follows by applying estimate (3.1) to control the term $M_{\mu}^{4 /(h+1)}\left\|\varphi_{\mu}^{w} u\right\|_{0}^{2}$ and by using (A.13) and Proposition A.9 to control the remainder terms.

Until now we reduced the proof of Theorem 2.4 to the proof of (3.7). We now want to reduce the proof of (3.7) to the proof of suitable inequalities on the rectangles of the partition $\left\{Q_{\mu \nu}\right\}$ constructed in Section 2 . 
Definition 3.3 Let $\bar{\mu}$ be a fixed arbitrary index in $\mathbb{N}$. If $\left\{Q_{\mu \nu}\right\}_{\mu \in \mathbb{N}, \nu=1, \ldots, N(\mu)}$ is the partition constructed in Section 2, we define

1) $I_{\bar{\mu}}=\left\{(\mu, \nu): \frac{13}{8} Q_{\mu \nu} \cap 2 Q_{\bar{\mu}} \neq \emptyset\right\} ;$

2) $I_{\bar{\mu}}^{e}=\left\{(\mu, \nu) \in I_{\bar{\mu}}\right.$ satisfying $\left.(2.6)\right\}$;

3) $I_{\bar{\mu}}^{s}=I_{\bar{\mu}} \backslash I_{\bar{\mu}}^{e}=\left\{(\mu, \nu) \in I_{\bar{\mu}}\right.$ satisfying only $\left.(2.5)\right\}$.

Note that $I_{\bar{\mu}}^{e} \cap I_{\bar{\mu}}^{s}=\emptyset$ and that $I_{\bar{\mu}}^{e} \cup I_{\bar{\mu}}^{s}=I_{\bar{\mu}}$.

Remark 3.4 One has $\rho_{\bar{\mu}} \sim \rho_{\mu} \lesssim \delta_{\mu \nu}$ for every $\mu \nu$ such that $(\mu, \nu) \in I_{\bar{\mu}}$. In fact, from $(13 / 8) Q_{\mu \nu} \cap 2 Q_{\bar{\mu}} \neq \emptyset$ it follows $2 Q_{\mu} \cap 2 Q_{\bar{\mu}} \neq \emptyset$ which in turn, by Remark 2.3, gives $M_{\mu} \sim M_{\bar{\mu}}$. The conclusion then follows by (2.16) and (2.17).

The next proposition clarifies the meaning of Definition 3.3. We, in fact, see that $I_{\bar{\mu}}$ describes the set of the indices that "count" when one microlocalizes (3.7) with $\mu=\bar{\mu}$ on the rectangles of the partition $\left\{Q_{\mu \nu}\right\}$.

Proposition 3.5 Let $p$ and $q$ be as in Theorem 2.4. Then, for $\bar{\mu} \in \mathbb{N}$, the estimate

$$
\left\|\left(\rho_{\bar{\mu}}^{2} p \psi_{\bar{\mu}}\right)^{w} u\right\|_{0}^{2} \gtrsim\left\|\left(\rho_{\bar{\mu}}^{2} q \psi_{\bar{\mu}}\right)^{w} u\right\|_{0}^{2}-\|u\|_{0}^{2}-\left(r_{\bar{\mu}, 2}^{w} u, u\right)_{L^{2}}, \quad \forall u \in \mathcal{S}\left(\mathbb{R}^{2}\right)
$$

with $\sum_{\bar{\mu} \in \mathbb{N}} \rho_{\bar{\mu}}^{-4} \varphi_{\bar{\mu}}^{w} r_{\bar{\mu}, 2}^{w} \varphi_{\bar{\mu}}^{w} \in \mathcal{L}\left(L^{2}\left(\mathbb{R}^{2}\right), L^{2}\left(\mathbb{R}^{2}\right)\right)$ holds if both the following estimates hold:

- for any $\mu \nu$ such that $(\mu, \nu) \in I_{\bar{\mu}}^{e}$ and for every $u \in \mathcal{S}\left(\mathbb{R}^{2}\right)$

$$
\begin{aligned}
& \left\|\left(\rho_{\bar{\mu}}^{2} p \psi_{\bar{\mu}} \psi_{\mu \nu}\right)^{w} \varphi_{\mu \nu}^{w} u\right\|_{0}^{2} \gtrsim\left\|\left(\rho_{\bar{\mu}}^{2} q \psi_{\bar{\mu}} \psi_{\mu \nu}\right)^{w} \varphi_{\mu \nu}^{w} u\right\|_{0}^{2}-\left\|\varphi_{\mu \nu}^{w} u\right\|_{0}^{2} \\
& \quad-\left\|\rho_{\bar{\mu}}^{2} \epsilon_{\mu \nu}^{2}\left(\left(1-\psi_{\bar{\mu}}\right) \psi_{\mu \nu}\right)^{w} \varphi_{\mu \nu}^{w} u\right\|_{0}^{2}-\left\|\rho_{\bar{\mu}}^{2} \epsilon_{\mu \nu}^{2}\left(1-\psi_{\mu \nu}\right)^{w} \varphi_{\mu \nu}^{w} u\right\|_{0}^{2} .
\end{aligned}
$$

- for any $\mu \nu$ such that $(\mu, \nu) \in I_{\bar{\mu}}^{s}$ and for every $u \in \mathcal{S}\left(\mathbb{R}^{2}\right)$

$$
\left\|\left(\rho_{\bar{\mu}}^{2} p \psi_{\bar{\mu}} \psi_{\mu \nu}\right)^{w} \varphi_{\mu \nu}^{w} u\right\|_{0}^{2} \gtrsim\left\|\left(\rho_{\bar{\mu}}^{2} q \psi_{\bar{\mu}} \psi_{\mu \nu}\right)^{w} \varphi_{\mu \nu}^{w} u\right\|_{0}^{2}-\left\|\varphi_{\mu \nu}^{w} u\right\|_{0}^{2} .
$$

Proof. By adding (3.9) to (3.10) and by using Theorem A.5, we see that it suffices to prove the following relations:

$$
\begin{aligned}
& \sum_{(\mu, \nu) \in I_{\bar{\mu}}}\left\|\left(\rho_{\bar{\mu}}^{2} q \psi_{\bar{\mu}} \psi_{\mu \nu}\right)^{w} \varphi_{\mu \nu}^{w} u\right\|_{0}^{2} \gtrsim\left\|\left(\rho_{\bar{\mu}}^{2} q \psi_{\bar{\mu}}\right)^{w} u\right\|_{0}^{2}-\|u\|_{0}^{2}, \quad \forall u \in \mathcal{S}\left(\mathbb{R}^{2}\right) \\
& \sum_{(\mu, \nu) \in I_{\bar{\mu}}^{e}}\left\|\rho_{\bar{\mu}}^{2} \epsilon_{\mu \nu}^{2}\left(1-\psi_{\mu \nu}\right)^{w} \varphi_{\mu \nu}^{w} u\right\|_{0}^{2} \lesssim\|u\|_{0}^{2}, \quad \forall u \in \mathcal{S}\left(\mathbb{R}^{2}\right)
\end{aligned}
$$


and

$$
\begin{gathered}
\sum_{(\mu, \nu) \in I_{\bar{\mu}}^{e}}\left\|\rho_{\bar{\mu}}^{2} \epsilon_{\mu \nu}^{2}\left(\psi_{\mu \nu}\left(1-\psi_{\bar{\mu}}\right)\right)^{w} \varphi_{\mu \nu}^{w} u\right\|_{0}^{2} \lesssim\left(R M_{\bar{\mu}}^{h}\right)^{4 /(h+1)}\left\|\left(1-\psi_{\bar{\mu}}\right)^{w} u\right\|_{0}^{2} \\
+\left\|\rho_{\bar{\mu}}^{2}\left(\left(p+R^{2}\right)\left(1-\psi_{\bar{\mu}}\right)\right)^{w} u\right\|_{0}^{2}+\|u\|_{0}^{2}, \quad \forall u \in \mathcal{S}\left(\mathbb{R}^{2}\right)
\end{gathered}
$$

Relations (3.11) and (3.12) are an immediate consequence of (A.14) and Proposition A.9. We proceed to prove (3.13). We first observe that

$$
\begin{array}{r}
\sum_{(\mu, \nu) \in I_{\bar{\mu}}^{e}}\left\|\rho_{\bar{\mu}}^{2} \epsilon_{\mu \nu}^{2}\left(\psi_{\mu \nu}\left(1-\psi_{\bar{\mu}}\right)\right)^{w} \varphi_{\mu \nu}^{w} u\right\|_{0}^{2} \lesssim \sum_{(\mu, \nu) \in I_{\bar{\mu}}^{e}}\left\|\rho_{\bar{\mu}}^{2} \epsilon_{\mu \nu}^{2} \varphi_{\mu \nu}^{w}\left(\psi_{\mu \nu}\left(1-\psi_{\bar{\mu}}\right)\right)^{w} u\right\|_{0}^{2} \\
+\sum_{(\mu, \nu) \in I_{\bar{\mu}}^{e}}\left\|\left[\rho_{\bar{\mu}}^{2} \epsilon_{\mu \nu}^{2}\left(\psi_{\mu \nu}\left(1-\psi_{\bar{\mu}}\right)\right)^{w}, \varphi_{\mu \nu}^{w}\right] u\right\|_{0}^{2}
\end{array}
$$

We now want to estimate the terms in the right hand side of (3.14). As concerns the first one, we can apply Proposition A.9 (in view of Remark 3.4) and get

$$
\sum_{(\mu, \nu) \in I_{\bar{\mu}}^{e}}\left\|\rho_{\bar{\mu}}^{2} \epsilon_{\mu \nu}^{2} \varphi_{\mu \nu}^{w}\left(\psi_{\mu \nu}\left(1-\psi_{\bar{\mu}}\right)\right)^{w} u\right\|_{0}^{2} \lesssim \sum_{(\mu, \nu) \in I_{\bar{\mu}}^{e}}\left\|\rho_{\bar{\mu}}^{2} \epsilon_{\mu \nu}^{2} \varphi_{\mu \nu}^{w}\left(1-\psi_{\bar{\mu}}\right)^{w} u\right\|_{0}^{2}+\|u\|_{0}^{2}
$$

Moreover, $\rho_{\bar{\mu}}^{4} \epsilon_{\mu \nu}^{4} \lesssim\left(R / M_{\bar{\mu}}\right)^{4 /(h+1)} M_{\mu}^{4} \delta_{\mu \nu}^{4 h} \lesssim\left(R M_{\bar{\mu}}^{h}\right)^{4 /(h+1)}$ then, applying (A.13),

$$
\sum_{(\mu, \nu) \in I_{\bar{\mu}}^{e}}\left\|\rho_{\bar{\mu}}^{2} \epsilon_{\mu \nu}^{2} \varphi_{\mu \nu}^{w}\left(1-\psi_{\bar{\mu}}\right)^{w} u\right\|_{0}^{2} \lesssim\left(R M_{\bar{\mu}}^{h}\right)^{4 /(h+1)}\left\|\left(1-\psi_{\bar{\mu}}\right)^{w} u\right\|_{0}^{2}
$$

Thus

$$
\sum_{(\mu, \nu) \in I_{\bar{\mu}}^{e}}\left\|\rho_{\bar{\mu}}^{2} \epsilon_{\mu \nu}^{2} \varphi_{\mu \nu}^{w}\left(\psi_{\mu \nu}\left(1-\psi_{\bar{\mu}}\right)\right)^{w} u\right\|_{0}^{2} \lesssim\left(R M_{\bar{\mu}}^{h}\right)^{4 /(h+1)}\left\|\left(1-\psi_{\bar{\mu}}\right)^{w} u\right\|_{0}^{2}+\|u\|_{0}^{2} .
$$

As for the second term in the right hand side of (3.14), we show that

$$
\begin{aligned}
\sum_{(\mu, \nu) \in I_{\bar{\mu}}^{e}}\left\|\left[\rho_{\bar{\mu}}^{2} \epsilon_{\mu \nu}^{2}\left(\psi_{\mu \nu}\left(1-\psi_{\bar{\mu}}\right)\right)^{w}, \varphi_{\mu \nu}^{w}\right] u\right\|_{0}^{2} \lesssim & \\
& \lesssim\left(\rho_{\bar{\mu}}^{2}\left(\left(p+R^{2}\right)\left(1-\psi_{\bar{\mu}}\right)\right)^{w} u, u\right)_{L^{2}}+\|u\|_{0}^{2}
\end{aligned}
$$

Note that the family $\left\{\varphi_{\mu \nu}\right\}$ is bounded in $S\left(1, g^{I I}\right)$ and that the family of positive functions $\left\{\rho_{\bar{\mu}}^{2} \epsilon_{\mu \nu}^{2} \psi_{\mu \nu}\left(1-\psi_{\bar{\mu}}\right)\right\}$ is bounded in $S\left(h_{I I}^{-2}, g^{I I}\right)$ with $\operatorname{supp} \varphi_{\mu \nu} \subseteq(9 / 8) Q_{\mu \nu}$ and $\operatorname{supp} \rho_{\bar{\mu}}^{2} \epsilon_{\mu \nu}^{2} \psi_{\mu \nu}\left(1-\psi_{\bar{\mu}}\right) \subseteq(13 / 8) Q_{\mu \nu} \subseteq(7 / 4) Q_{\mu \nu}$. 
Thus (A.25) applies yielding

$$
\begin{aligned}
& \sum_{(\mu, \nu) \in I_{\bar{\mu}}^{e}}\left\|\left[\varphi_{\mu \nu}^{w}, \rho_{\bar{\mu}}^{2} \epsilon_{\mu \nu}^{2}\left(\psi_{\mu \nu}\left(1-\psi_{\bar{\mu}}\right)\right)^{w}\right] u\right\|_{0}^{2} \lesssim \\
& \quad \lesssim \sum_{(\mu, \nu) \in I_{\bar{\mu}}^{e}}\left(\psi_{\mu \nu}^{w}\left(\rho_{\bar{\mu}}^{2} \epsilon_{\mu \nu}^{2} \psi_{\mu \nu}\left(1-\psi_{\bar{\mu}}\right)\right)^{w} \psi_{\mu \nu}^{w} u, u\right)_{L^{2}}+\|u\|_{0}^{2} .
\end{aligned}
$$

Moreover, from (A.24) it follows

$$
\begin{aligned}
\sum_{(\mu, \nu) \in I_{\bar{\mu}}^{e}} \psi_{\mu \nu}^{w}\left(\rho_{\bar{\mu}}^{2} \epsilon_{\mu \nu}^{2} \psi_{\mu \nu}\left(1-\psi_{\bar{\mu}}\right)\right)^{w} \psi_{\mu \nu}^{w}= & \\
= & \left(\sum_{(\mu, \nu) \in I_{\bar{\mu}}^{e}} \psi_{\mu \nu}^{3} \rho_{\bar{\mu}}^{2} \epsilon_{\mu \nu}^{2}\left(1-\psi_{\bar{\mu}}\right)\right)^{w}+r_{\bar{\mu}, 3}^{w}
\end{aligned}
$$

with $r_{\bar{\mu}, 3}^{w} \in \mathcal{L}\left(L^{2}\left(\mathbb{R}^{2}\right), L^{2}\left(\mathbb{R}^{2}\right)\right)$ having norm bounded uniformly in $\bar{\mu}$. Let us now define $J_{\bar{\mu}}=\left\{\mu^{\prime} \in \mathbb{N}: \exists(\mu, \nu) \in I_{\bar{\mu}},(13 / 8) Q_{\mu \nu} \cap(9 / 8) Q_{\mu^{\prime}} \neq \emptyset\right\}$ and observe that, since $\operatorname{supp} \psi_{\mu \nu}^{3} \subseteq(13 / 8) Q_{\mu \nu}$, we have

$$
\sum_{(\mu, \nu) \in I_{\bar{\mu}}^{e}} \psi_{\mu \nu}^{3} \rho_{\bar{\mu}}^{2} \epsilon_{\mu \nu}^{2}\left(1-\psi_{\bar{\mu}}\right)=\sum_{(\mu, \nu) \in I_{\bar{\mu}}^{e}} \psi_{\mu \nu}^{3} \rho_{\bar{\mu}}^{2} \epsilon_{\mu \nu}^{2}\left(1-\psi_{\bar{\mu}}\right)\left(\sum_{\mu^{\prime} \in J_{\bar{\mu}}} \varphi_{\mu^{\prime}}^{2}\right) .
$$

From Remark 2.9 one has, for $(\mu, \nu) \in I_{\bar{\mu}}^{e}$,

$$
\psi_{\mu, \nu}^{3} \rho_{\bar{\mu}}^{2} \epsilon_{\mu \nu}^{2}\left(1-\psi_{\bar{\mu}}\right) \lesssim \rho_{\bar{\mu}}^{2}\left(p+R^{2}\right)\left(1-\psi_{\bar{\mu}}\right) .
$$

Hence by the finite overlapping properties and (3.19)

$$
\sum_{(\mu, \nu) \in I_{\bar{\mu}}^{e}} \psi_{\mu \nu}^{3} \rho_{\bar{\mu}}^{2} \epsilon_{\mu \nu}^{2}\left(1-\psi_{\bar{\mu}}\right) \lesssim \sum_{\mu^{\prime} \in J_{\bar{\mu}}} \rho_{\bar{\mu}}^{2}\left(p+R^{2}\right)\left(1-\psi_{\bar{\mu}}\right) \varphi_{\mu^{\prime}}^{2}
$$

We can now apply the Fefferman-Phong inequality in the class $S\left(h_{I I}^{-2}, g^{I I}\right)$ and get

$$
\begin{aligned}
\left(\left(\sum_{(\mu, \nu) \in I_{\bar{\mu}}^{e}} \psi_{\mu \nu}^{3} \rho_{\bar{\mu}}^{2} \epsilon_{\mu \nu}^{2}\left(1-\psi_{\bar{\mu}}\right)\right)^{w} u, u\right)_{L^{2}} \lesssim & \\
& \lesssim\left(\left(\sum_{\mu^{\prime} \in J_{\bar{\mu}}} \varphi_{\mu^{\prime}}^{2} \rho_{\bar{\mu}}^{2}\left(p+R^{2}\right)\left(1-\psi_{\bar{\mu}}\right)\right)^{w} u, u\right)_{L^{2}}+\|u\|_{0}^{2} .
\end{aligned}
$$

Finally, since (again by finite overlapping)

$$
\sum_{\mu^{\prime} \in J_{\bar{\mu}}} \varphi_{\mu^{\prime}}^{2} \rho_{\bar{\mu}}^{2}\left(p+R^{2}\right)\left(1-\psi_{\bar{\mu}}\right) \lesssim \rho_{\bar{\mu}}^{2}\left(p+R^{2}\right)\left(1-\psi_{\bar{\mu}}\right),
$$

we can again apply the Fefferman-Phong inequality in the class $S\left(h_{I}^{-2}, g^{I}\right)$ and obtain the inequality (3.16) by (3.17), (3.18) and (3.20). An application of (3.15) and (3.16) to (3.14) completes the proof. 
Proof of Theorem 2.4. We use Propositions 3.2 and 3.5 and reduce the proof of Theorem 2.4 to the proof of estimates (3.9) and (3.10).

In order to prove (3.9), let us first observe that, for every $(\mu, \nu) \in I_{\bar{\mu}}^{e}$, the following inequality holds for $u \in \mathcal{S}\left(\mathbb{R}^{2}\right)$

$$
\begin{aligned}
& \left(\left(\rho_{\bar{\mu}}^{2}\left(p+R^{2}\right) \psi_{\bar{\mu}} \psi_{\mu \nu}+\left(1-\psi_{\mu \nu}\right) \rho_{\bar{\mu}}^{2} \epsilon_{\mu \nu}^{2}\right)^{w} u, u\right)_{L^{2}} \geq \\
& \quad \geq-\left(\rho_{\bar{\mu}}^{2} \epsilon_{\mu \nu}^{2}\left(\psi_{\mu \nu}\left(1-\psi_{\bar{\mu}}\right)\right)^{w} u, u\right)_{L^{2}}+\epsilon_{\mu \nu}^{2} \rho_{\bar{\mu}}^{2}\|u\|_{0}^{2}-C\|u\|_{0}^{2} .
\end{aligned}
$$

If, in fact, we consider the family of constant metrics

$$
g_{\mu \nu}(y, \eta)=\frac{|y|^{2}}{\delta_{\mu \nu}^{2}}+\frac{|\eta|^{2}}{\epsilon_{\mu \nu}^{2}},
$$

by (2.17), Proposition 2.13 and Remark 3.4 one has, for every $(\mu, \nu) \in I_{\bar{\mu}}^{e}$, that

$\rho_{\bar{\mu}}^{2}\left(p+R^{2}\right) \psi_{\bar{\mu}} \psi_{\mu \nu}+\left(1-\psi_{\mu \nu}\right) \rho_{\bar{\mu}}^{2} \epsilon_{\mu \nu}^{2}, \rho_{\bar{\mu}}^{2} \epsilon_{\mu \nu}^{2}$ and $\rho_{\bar{\mu}}^{2} \epsilon_{\mu \nu}^{2} \psi_{\mu \nu}\left(1-\psi_{\bar{\mu}}\right) \in S\left(h_{g_{\mu \nu}}^{-2}, g_{\mu \nu}\right)$

with seminorms independent of $\mu, \nu, \bar{\mu}$.

Moreover, from Remark 2.9 it follows that

$$
\rho_{\bar{\mu}}^{2}\left(p+R^{2}\right) \psi_{\bar{\mu}} \psi_{\mu \nu}+\left(1-\psi_{\mu \nu}\right) \rho_{\bar{\mu}}^{2} \epsilon_{\mu \nu}^{2} \geq \rho_{\bar{\mu}}^{2} \epsilon_{\mu \nu}^{2}-\rho_{\bar{\mu}}^{2} \epsilon_{\mu \nu}^{2} \psi_{\mu \nu}\left(1-\psi_{\bar{\mu}}\right) .
$$

Thus (3.21) follows by the Fefferman-Phong inequality.

If we observe that $R^{2} \rho_{\bar{\mu}}^{2} \psi_{\bar{\mu}} \psi_{\mu \nu}$ is in $S\left(1, g_{\mu \nu}\right)$ with seminorms independent of $\bar{\mu}, \mu, \nu$, we then get

$$
\begin{gathered}
\left\|\left(\rho_{\bar{\mu}}^{2} p \psi_{\mu} \psi_{\mu \nu}\right)^{w} u\right\|_{0}^{2} \geq \epsilon_{\mu \nu}^{4} \rho_{\bar{\mu}}^{4}\|u\|_{0}^{2}-C^{\prime}\|u\|_{0}^{2}-\left\|\rho_{\bar{\mu}}^{2} \epsilon_{\mu \nu}^{2}\left(\left(1-\psi_{\bar{\mu}}\right) \psi_{\mu \nu}\right)^{w} u\right\|_{0}^{2} \\
-\left\|\rho_{\bar{\mu}}^{2} \epsilon_{\mu \nu}^{2}\left(1-\psi_{\mu \nu}\right)^{w} u\right\|_{0}^{2}, \forall(\mu, \nu) \in I_{\bar{\mu}}^{e}, \forall u \in \mathcal{S}\left(\mathbb{R}^{2}\right) .
\end{gathered}
$$

Proceeding as in the proof of Proposition 2.14 we also have

$$
\delta_{\mu \nu}^{2} q \psi_{\bar{\mu}} \psi_{\mu \nu} \in S\left(h_{I I}^{-2}, g^{I I}\right)
$$

uniformly in $\bar{\mu}, \mu, \nu$ and this yields

$$
\left\|\rho_{\bar{\mu}}^{2}\left(q \psi_{\bar{\mu}} \psi_{\mu \nu}\right)^{w} u\right\|_{0}^{2} \lesssim \epsilon_{\mu \nu}^{4} \rho_{\bar{\mu}}^{4}\|u\|_{0}^{2}, \quad \forall(\mu, \nu) \in I_{\bar{\mu}}^{e}, \forall u \in \mathcal{S}\left(\mathbb{R}^{2}\right) .
$$

Finally, from (3.22) and (3.23), (3.9) readily follows.

For what concerns estimate (3.10), note that by (2.11) and (2.12) we have $\delta_{\mu \nu} \epsilon_{\mu \nu} \sim 1$ when $(\mu, \nu) \in I_{\bar{\mu}}^{s}$. Hence (3.10) follows by $L^{2}$-continuity and this concludes the proof. 


\section{A. Appendix}

Let us fix a sequence $\left\{\left(x_{k}, \xi_{k}\right)\right\}_{k \in \mathbb{N}}$ of points in the phase space $T^{*} \mathbb{R}^{n} \simeq$ $\mathbb{R}_{x}^{n} \times \mathbb{R}_{\xi}^{n}$ and two sequences $\left\{\delta_{k}\right\}_{k \in \mathbb{N}},\left\{\epsilon_{k}\right\}_{k \in \mathbb{N}}$ of real positive numbers. We denote by $B_{k}$ the rectangles defined by

$$
B_{k}=\left\{(x, \xi) \in T^{*} \mathbb{R}^{n}|| x_{j}-x_{k, j}\left|\leq \frac{\delta_{k}}{2 \sqrt{n}},\right| \xi_{j}-\xi_{k, j} \mid \leq \frac{\epsilon_{k}}{2 \sqrt{n}}, j=1, \ldots, n\right\}
$$

and assume that $\left\{B_{k}\right\}_{k \in \mathbb{N}}$ is a partition of $T^{*} \mathbb{R}^{n}$, i.e.

$$
\bigcup_{k \in \mathbb{N}} B_{k}=T^{*} \mathbb{R}^{n} \quad \text { and } \quad \stackrel{\circ}{B}_{k} \cap \stackrel{\circ}{B}_{k^{\prime}}=\emptyset \quad \text { for every } k, k^{\prime} \in \mathbb{N}, k \neq k^{\prime} .
$$

Consider the functions $\delta, \epsilon: \mathbb{R}_{x}^{n} \times \mathbb{R}_{\xi}^{n} \longrightarrow \mathbb{R}_{+}$defined by

$$
\delta(x, \xi)=\delta_{\gamma}, \quad \epsilon(x, \xi)=\epsilon_{\gamma} \quad \text { where } \gamma=\min \left\{k \in \mathbb{N}:(x, \xi) \in B_{k}\right\}
$$

and notice that $\delta(x, \xi)=\delta_{k}, \epsilon(x, \xi)=\epsilon_{k}$ when $(x, \xi) \in \stackrel{\circ}{B}_{k}$.

Remark A.1 If the partition is such that $\delta_{k} \sim \delta_{k^{\prime}}$ and $\epsilon_{k} \sim \epsilon_{k^{\prime}}$ whenever $2 B_{k} \cap 2 B_{k^{\prime}} \neq \emptyset$ then

$$
(x, \xi) \in B_{k} \Rightarrow \delta(x, \xi) \sim \delta_{k}, \quad \epsilon(x, \xi) \sim \epsilon_{k} .
$$

For any $(x, \xi) \in \mathbb{R}_{x}^{n} \times \mathbb{R}_{\xi}^{n}$, we consider a positive definite quadratic form $g_{(x, \xi)}$ of $(t, \tau) \in \mathbb{R}^{2 n}$ as follows

$$
g_{(x, \xi)}(t, \tau)=\frac{|t|^{2}}{\delta(x, \xi)^{2}}+\frac{|\tau|^{2}}{\epsilon(x, \xi)^{2}} .
$$

It is important to point out that the Riemannian metric $g:(x, \xi) \longrightarrow g_{(x, \xi)}$ is not, in general, continuous on the boundary of each $B_{k}$, but that causes no problem as shown in [14].

In this setting, for any $(x, \xi) \in \mathbb{R}_{x}^{n} \times \mathbb{R}_{\xi}^{n}$ the dual metric $g^{\sigma}$ of $g$ (with respect to the canonical symplectic form $\sigma$ on $T^{*} \mathbb{R}^{n}$ ) is given by

$$
g_{(x, \xi)}^{\sigma}(t, \tau)=\epsilon(x, \xi)^{2}|t|^{2}+\delta(x, \xi)^{2}|\tau|^{2}, \quad \forall(t, \tau) \in \mathbb{R}^{2 n},
$$

while the Planck function $h_{g}: T^{*} \mathbb{R}^{n} \longrightarrow \mathbb{R}_{+}$is given by

$$
h_{g}(x, \xi)=\delta(x, \xi)^{-1} \epsilon(x, \xi)^{-1} .
$$

The following proposition establishes sufficient conditions under which $g$ is a Weyl-Hörmander metric. 
Proposition A.2 Fix a positive integer $h$ and a real positive number $R \geq 1$. Suppose that, for the partition $\left\{B_{k}\right\}_{k \in \mathbb{N}}$ defined in (A.1), the following conditions hold:

$$
\begin{gathered}
\epsilon_{k} \sim(R+|\xi|) \delta_{k}^{h}, \quad \forall(x, \xi) \in B_{k} ; \\
R^{1 /(h+1)}(R+|\xi|)^{-1 /(h+1)} \leq \delta_{k} \leq 1, \quad \forall(x, \xi) \in B_{k} \\
\text { If } 2 B_{k} \cap 2 B_{k^{\prime}} \neq \emptyset \Longrightarrow \delta_{k} \sim \delta_{k^{\prime}} \text { and } \epsilon_{k} \sim \epsilon_{k^{\prime}} .
\end{gathered}
$$

Then $g$ defined by (A.2) is a Weyl-Hörmander metric provided $R$ is chosen large enough, i.e. $g$ is slowly varying and $\sigma$-temperate (see [9]). Moreover, (A.5) determines an a priori bounded number $K=K(n)$ of overlappings among the rectangles $2 B_{k}$. Namely, one has $\left|J_{k}\right| \leq K$ for every $k \in \mathbb{N}$, where

$$
J_{k}=\left\{k^{\prime} \in \mathbb{N}: 2 B_{k^{\prime}} \cap 2 B_{k} \neq \emptyset\right\}
$$

and $\left|J_{k}\right|$ denotes its cardinality.

Proof. We start off by proving that $g$ is a slowly varying metric. Suppose that $g_{(y, \eta)}(x-y, \xi-\eta) \leq 1 /(4 n)$ with $(x, \xi) \in B_{k}$. Then $(x, \xi) \in 2 B_{\gamma}$ with $\gamma=\min \left\{\omega \in \mathbb{N}:(y, \eta) \in B_{\omega}\right\}$. This gives, in view of Remark A.1 and (A.5), the existence of positive constants $c_{1}, c_{2}$ such that

$$
c_{1} g_{(x, \xi)}(t, \tau) \leq g_{(y, \eta)}(t, \tau) \leq c_{2} g_{(x, \xi)}(t, \tau), \quad \forall(t, \tau) \in \mathbb{R}^{2 n},
$$

for such $(x, \xi)$. Hence, $g$ is slowly varying.

From (A.3) and (A.4) it follows, if $R$ is chosen large enough, $h_{g}(x, \xi)<1$ for any $(x, \xi) \in \mathbb{R}^{2 n}$, whence $g_{(x, \xi)}(t, \tau) \leq g_{(x, \xi)}^{\sigma}(t, \tau)$. It remains to show that there exist a positive constant $c$ and a positive integer $Q$ for which

$$
g_{(x, \xi)}(t, \tau) \leq c g_{(y, \eta)}(t, \tau)\left(1+g_{(x, \xi)}^{\sigma}(y-x, \eta-\xi)\right)^{Q},
$$

whenever $(x, \xi),(y, \eta) \in \mathbb{R}^{2 n}$. Actually we prove the following equivalent inequality

$$
g_{(y, \eta)}^{\sigma}(t, \tau) \leq c g_{(x, \xi)}^{\sigma}(t, \tau)\left(1+g_{(x, \xi)}^{\sigma}(y-x, \eta-\xi)\right)^{h+1} .
$$

We first observe that, from Remark A.1 it follows

$$
\sup _{(t, \tau) \in \mathbb{R}^{2 n}} \frac{g_{(y, \eta)}^{\sigma}(t, \tau)}{g_{(x, \xi)}^{\sigma}(t, \tau)} \sim \max \left\{\frac{\epsilon_{k^{\prime}}^{2}}{\epsilon_{k}^{2}}, \frac{\delta_{k^{\prime}}^{2}}{\delta_{k}^{2}}\right\}, \quad \forall(x, \xi) \in B_{k}, \forall(y, \eta) \in B_{k^{\prime}} ;
$$

therefore, if $g_{(y, \eta)}(x-y, \xi-\eta) \leq 1 /(4 n)$, inequality (A.7) easily follows from the slowly varying property just because the right hand side of (A.8) 
is bounded. When $g_{(y, \eta)}(x-y, \xi-\eta)>1 /(4 n)$, again by using Remark A.1, we have two cases:

$$
\text { either }|\xi-\eta|^{2} \gtrsim \epsilon_{k^{\prime}}^{2} \quad \text { or } \quad|x-y|^{2} \gtrsim \delta_{k^{\prime}}^{2}
$$

Assuming $|\xi-\eta|^{2} \gtrsim \epsilon_{k^{\prime}}^{2}$, we get

$$
\left(1+g_{(x, \xi)}^{\sigma}(y-x, \eta-\xi)\right) \epsilon_{k}^{2} \gtrsim \epsilon_{k}^{2} \delta_{k}^{2}|\xi-\eta|^{2} \gtrsim \epsilon_{k^{\prime}}^{2} .
$$

If $|\xi-\eta| \geq|\xi| / 2$, from (A.4) it follows

$$
\begin{aligned}
\left(1+g_{(x, \xi)}^{\sigma}(y-x, \eta-\xi)\right) \delta_{k}^{2} & \gtrsim\left(1+\delta_{k}^{2}|\xi|^{2}\right) \delta_{k}^{2} \geq \frac{1}{R^{2}} \delta_{k}^{4}(R+|\xi|)^{2} \\
& \geq \frac{1}{R^{2}} \frac{R^{4 /(h+1)}}{(R+|\xi|)^{4 /(h+1)}}(R+|\xi|)^{2} \gtrsim 1 \gtrsim \delta_{k^{\prime}}^{2} .
\end{aligned}
$$

Suppose now that $|\xi-\eta| \leq|\xi| / 2$, in view of (A.3) and (A.9) we have

$$
\begin{aligned}
\left(1+g_{(x, \xi)}^{\sigma}(y-x, \eta-\xi)\right) \delta_{k}^{2 h} & \gtrsim\left(\left(1+g_{(x, \xi)}^{\sigma}(y-x, \eta-\xi)\right) \epsilon_{k}^{2}\right)(R+|\xi|)^{-2} \\
& \gtrsim \epsilon_{k^{\prime}}^{2}(R+|\eta|)^{-2} \gtrsim \delta_{k^{\prime}}^{2 h}
\end{aligned}
$$

Inequality (A.7) follows in this case from (A.8). The case $|x-y|^{2} \gtrsim \delta_{k^{\prime}}^{2}$ is achieved similarly.

It remains to show the existence of an a priori bounded number of overlappings for the covering $\left\{B_{k}\right\}_{k \in \mathbb{N}}$. Suppose that $2 B_{k^{\prime}} \cap 2 B_{k} \neq \emptyset$. Then there exists $(x, \xi) \in 2 B_{k^{\prime}} \cap 2 B_{k}$, and from (A.5) it follows, for a constant $c>0$ independent of $k, k^{\prime}, \delta_{k} / c \leq \delta_{k^{\prime}} \leq c \delta_{k}, \epsilon_{k} / c \leq \epsilon_{k^{\prime}} \leq c \epsilon_{k}$. Upon denoting by $\left(x_{k}, \xi_{k}\right)$ the center of the rectangle $B_{k}$, one has, for every $(y, \eta) \in 2 B_{k^{\prime}}$,

$$
\left|y_{i}-x_{k, i}\right| \leq\left|y_{i}-x_{i}\right|+\left|x_{i}-x_{k, i}\right| \leq 2 \delta_{k^{\prime}}+\delta_{k} \leq(1+2 c) \delta_{k}, \quad i=1, \ldots, n,
$$

and, in a similar way, $\left|\eta_{i}-\xi_{k, i}\right| \leq(1+2 c) \epsilon_{k}, i=1, \ldots, n$. Therefore one gets

$$
\bigcup_{k^{\prime} \in J_{k}} B_{k^{\prime}} \subseteq(1+2 c) 2 \sqrt{n} B_{k}
$$

whence

$$
\sum_{k^{\prime} \in J_{k}} m\left(B_{k^{\prime}}\right)=m\left(\bigcup_{k^{\prime} \in J_{k}} B_{k^{\prime}}\right) \leq m\left((1+2 c) 2 \sqrt{n} B_{k}\right)
$$

where $m(A)$ denotes the Lebesgue measure of a set $A$, and from this the conclusion follows since $m\left(B_{k^{\prime}}\right) \sim \epsilon_{k}^{n} \delta_{k}^{n}$ for each $k^{\prime} \in J_{k}$ and $m\left(B_{k}\right) \sim \epsilon_{k}^{n} \delta_{k}^{n}$. 
In the sequel we assume that the hypotheses of Proposition A.2 hold for the partition defined by (A.1). As a consequence, $g$ is slowly varying and $\sigma$-temperate, and an easy check shows that the Planck function $h_{g}$ is an admissible weight for $g$.

We now define the class $S\left(h_{g}^{s}, g\right)(s \in \mathbb{R})$ as follows.

Definition A.3 Let $j$ be a non negative integer and $(x, \xi)$ be a point of $\mathbb{R}_{x}^{n} \times \mathbb{R}_{\xi}^{n} ;$ define, for any smooth function $b \in C^{\infty}\left(\mathbb{R}_{x}^{n} \times \mathbb{R}_{\xi}^{n}\right)$,

$$
|b|_{j}^{g}(x, \xi)=\max _{|\alpha+\beta| \leq j} \delta(x, \xi)^{|\alpha|} \epsilon(x, \xi)^{|\beta|}\left|D_{x}^{\alpha} D_{\xi}^{\beta} b(x, \xi)\right| .
$$

We denote by $S\left(h_{g}^{s}, g\right)(s \in \mathbb{R})$ the class of the functions $b \in C^{\infty}\left(\mathbb{R}^{n} \times \mathbb{R}^{n}\right)$ such that, for any integer $l \geq 0$,

$$
\|b\|_{\left(l, h_{g}^{s}\right)}=\sup _{(x, \xi) \in \mathbb{R}^{2 n}} h_{g}(x, \xi)^{-s}|b|_{l}^{g}(x, \xi)<+\infty .
$$

It turns out that $S\left(h_{g}^{s}, g\right)$ is a Fréchet space with the topology induced by the seminorms defined in (A.11).

As a consequence of Remark A.1, we have

$$
\|b\|_{\left(l, h_{g}^{s}\right)} \sim \sup _{k \in \mathbb{N}} \max _{\substack{(x, \xi) \in B_{k} \\|\alpha+\beta| \leq l}} \delta_{k}^{s+|\alpha|} \epsilon_{k}^{s+|\beta|}\left|D_{x}^{\alpha} D_{\xi}^{\beta} b(x, \xi)\right| .
$$

By proceeding as in [2] we get a partition of unity related to the covering $\left\{B_{k}\right\}_{k \in \mathbb{N}}$.

Proposition A.4 There exist two families of smooth functions $\left\{\varphi_{k}\right\}_{k \in \mathbb{N}}$, $\left\{\psi_{k}\right\}_{k \in \mathbb{N}}$ defined in $\mathbb{R}_{x}^{n} \times \mathbb{R}_{\xi}^{n}$, uniformly bounded in the space $S(1, g)$, with the following properties:

1) for every $j=0,1, \ldots$, there exists a positive constant $C_{j}$ independent of $R$, such that

$$
\left\|\varphi_{k}\right\|_{(j, 1)}+\left\|\psi_{k}\right\|_{(j, 1)} \leq C_{j}, \quad \forall k \in \mathbb{N}
$$

2) for any $k \in \mathbb{N}$,

$$
\begin{gathered}
0 \leq \varphi_{k}(x, \xi) \leq 1, \quad 0 \leq \psi_{k}(x, \xi) \leq 1, \quad \forall(x, \xi) \in \mathbb{R}_{x}^{n} \times \mathbb{R}_{\xi}^{n} \\
\operatorname{supp} \varphi_{k} \subseteq \frac{9}{8} B_{k}, \quad \operatorname{supp} \psi_{k} \subseteq \frac{13}{8} B_{k}, \quad \psi_{k} \equiv 1 \quad \text { on } \frac{3}{2} B_{k} ;
\end{gathered}
$$

3) for every $(x, \xi) \in \mathbb{R}_{x}^{n} \times \mathbb{R}_{\xi}^{n}$, one has $\sum_{k \in \mathbb{N}} \varphi_{k}(x, \xi)^{2} \equiv 1$.

In what follows, we refer to the pair $\left\{\varphi_{k}\right\}_{k \in \mathbb{N}},\left\{\psi_{k}\right\}_{k \in \mathbb{N}}$ as a partition of unity associated with the covering $\left\{B_{k}\right\}_{k \in \mathbb{N}}$. 
The following theorem is the main result we use to reduce the proof of inequality (1.7) to microlocal estimates on suitable rectangles of the cotangent space.

Theorem A.5 Let $\left\{\varphi_{k}\right\}_{k \in \mathbb{N}},\left\{\psi_{k}\right\}_{k \in \mathbb{N}}$ be a partition of unity associated with $\left\{B_{k}\right\}_{k \in \mathbb{N}}$. Then

1) there exists a constant $c_{1}>0$ independent of $R$ such that

$$
\sum_{k \in \mathbb{N}}\left\|\varphi_{k}^{w} u\right\|_{0}^{2} \leq c_{1}\|u\|_{0}^{2}, \quad \forall u \in \mathcal{S}\left(\mathbb{R}^{n}\right)
$$

2) for any $p \in S\left(h_{g}^{-2}, g\right)$, there exist positive constants $c_{2}, c_{2}^{\prime}$ such that

$$
\left\|p^{w} u\right\|_{0}^{2} \leq c_{2} \sum_{k \in \mathbb{N}}\left\|\left(p \psi_{k}\right)^{w} \varphi_{k}^{w} u\right\|_{0}^{2}+c_{2}^{\prime}\|u\|_{0}^{2}, \quad \forall u \in \mathcal{S}\left(\mathbb{R}^{n}\right),
$$

with $c_{2}$ independent of $R$.

Furthermore, if $p \in S\left(h_{g}^{-2}, g\right)$ is a real non-negative symbol, then, for suitable positive constants $c_{3}, c_{3}^{\prime}$, we have

$$
c_{3}\left\|p^{w} u\right\|_{0}^{2}+c_{3}^{\prime}\|u\|_{0}^{2} \geq \sum_{k \in \mathbb{N}}\left\|\left(p \psi_{k}\right)^{w} \varphi_{k}^{w} u\right\|_{0}^{2}, \quad \forall u \in \mathcal{S}\left(\mathbb{R}^{n}\right)
$$

Moreover, if $\delta_{k}^{-2+|\alpha|} \epsilon_{k}^{-2+|\beta|}\left|\partial_{x}^{\alpha} \partial_{\xi}^{\beta} p_{\mid B_{k}}\right|$ are uniformly bounded in $R$, for any rectangle $B_{k}$ having center, $\left(x_{k}, \xi_{k}\right)$, satisfying $\left|\xi_{k}\right| \geq 2 R$, then $c_{3}$ can be chosen not depending on $R$.

The proof of this proposition requires some preliminary results we give below.

Lemma A.6 Let $\left\{a_{k}\right\}_{k \in \mathbb{N}}$ be a sequence of symbols in $S\left(h_{g}^{s}, g\right)$ for any $s \in \mathbb{R}$ and assume that, for any $j=0,1, \ldots$, there exists a constant $C_{j}>0$ such that

$$
\sum_{k \in \mathbb{N}}\left|a_{k}\right|_{j}^{g}(x, \xi) \leq C_{j} h_{g}(x, \xi)^{s}, \quad \forall(x, \xi) \in \mathbb{R}_{x}^{n} \times \mathbb{R}_{\xi}^{n} .
$$

Then one has $\sum_{k \in \mathbb{N}} a_{k} \in S\left(h_{g}^{s}, g\right)$ and, for every $u \in \mathcal{S}\left(\mathbb{R}^{n}\right)$,

$$
\sum_{k \in \mathbb{N}} a_{k}^{w} u=\left(\sum_{k \in \mathbb{N}} a_{k}\right)^{w} u
$$

where $\sum_{k \in \mathbb{N}} a_{k}^{w} u$ denotes the $\mathcal{S}\left(\mathbb{R}^{n}\right)$ - limit of $\left\{\sum_{k \leq N} a_{k}^{w} u\right\}_{N \in \mathbb{N}}$ as $N$ tends to $+\infty$. Furthermore, if $s=0$ then $\left(\sum_{k \in \mathbb{N}} a_{k}\right)^{\bar{w}}$ gives rise to a linear continuous operator from $L^{2}\left(\mathbb{R}^{n}\right)$ to $L^{2}\left(\mathbb{R}^{n}\right)$. 
Proof. As a consequence of (A.16), $\left\{\sum_{k \leq N} a_{k}\right\}_{N \in \mathbb{N}}$ is a bounded sequence in $C^{\infty}\left(\mathbb{R}^{2 n}\right)$. In fact, for any compact set $K$ of $\mathbb{R}^{2 n}, K \subset \bigcup_{\mu=1}^{N_{K}} B_{\mu}$ and this gives

$$
\delta(x, \xi) \sim c_{K}, \epsilon(x, \xi) \sim C_{K} \quad \text { for any }(x, \xi) \in K .
$$

From standard arguments we then get that $\left\{\sum_{k \leq N} a_{k}\right\}_{N \in \mathbb{N}}$ is a convergent sequence in $C^{\infty}$ if it converges pointwise. To this purpose, note that, by Theorem 14.4 of [17], it has a subsequence converging to a smooth function $a(x, \xi)$. By (A.16), choosing $j=0$, we then have that $\left\{\sum_{k \leq N} a_{k}\right\}_{N \in \mathbb{N}}$ converges pointwise to $a$.

As a consequence, $a$ is in $S\left(h_{g}^{s}, g\right)$ and $\left\{\sum_{k \leq N} a_{k}^{w} u\right\}$ is a bounded sequence in $\mathcal{S}\left(\mathbb{R}^{n}\right)$. Again, if we prove that it converges to $a^{w} u$ pointwise we get convergence in $\mathcal{S}\left(\mathbb{R}^{n}\right)$. Since the pointwise convergence is an immediate consequence of the Lebesgue Theorem the proof is thus complete.

The following lemma is proved in [9, Theorem 4.2, pages 390-391].

Lemma A.7 Suppose that $q_{k} \in C_{0}^{\infty}\left(\frac{7}{4} B_{k}\right)$ and $q_{k^{\prime}} \in C_{0}^{\infty}\left(\frac{7}{4} B_{\gamma}\right)$ with $k, k^{\prime} \in \mathbb{N}$. For $N=0,1, \ldots$, set

$$
T_{N}\left(q_{k}, q_{k^{\prime}}\right)(x, \xi)=\sum_{j<N} \frac{1}{j !}\left(\frac{1}{2 i} \sigma\left(D_{x}, D_{\xi} ; D_{y}, D_{\eta}\right)\right)^{j} q_{k}(x, \xi) q_{k^{\prime}}(y, \eta) \mid \begin{gathered}
y=x \\
\eta=\xi
\end{gathered}
$$

where $\sigma$ is the standard symplectic 2-form, i.e.

$$
\sigma((x, \xi) ;(y, \eta))=\langle\xi, y\rangle-\langle x, \eta\rangle \quad \text { for every }(x, \xi),(y, \eta) \in \mathbb{R}^{2 n} .
$$

Then, for any $j, N=0,1, \ldots$, for any $s \geq 0$ and any $r, t$, there exist positive constants $c_{j}$ and $l$ independent of $R$ such that

$$
\begin{aligned}
\mid q_{k} \sharp q_{k^{\prime}} & -\left.T_{N}\left(q_{k}, q_{k^{\prime}}\right)\right|_{j} ^{g}(x, \xi) \leq \\
& \leq c_{j} h_{g}(x, \xi)^{r+t+N}\left(2+d_{k}(x, \xi)+d_{k^{\prime}}(x, \xi)\right)^{-s}\left\|q_{k}\right\|_{\left(l, h_{g}^{r}\right)}\left\|q_{k^{\prime}}\right\|_{\left(l, h_{g}^{t}\right)},
\end{aligned}
$$

where $a \sharp b$ is the Weyl symbol of the composition $a^{w} b^{w}\left(\right.$ i.e. $\left.(a \sharp b)^{w}=a^{w} b^{w}\right)$ and $d_{k}$ is defined by

$$
d_{k}(x, \xi)=\inf _{(y, \eta) \in \frac{15}{8} B_{k}} g_{(x, \xi)}^{\sigma}(x-y, \xi-\eta)
$$

The next lemma, due to Fujiwara (see [7, Proposition A2.4] or [9, Thm. 5.3]), is used in the proof of Proposition A.9 which is the main tool we need to prove Theorem A.5. 
Lemma A.8 Let $s \in \mathbb{R}$ be such that $s>(Q+1) n$ (where $Q$ is the constant appearing in (A.6)) and let $A$ be a positive number. Define

$$
d_{\nu k}=\inf _{(x, \xi) \in \frac{15}{8} B_{\nu}} d_{k}(x, \xi)
$$

Then there exists a constant $C>0$ independent of $R$ such that, for any $(x, \xi) \in \mathbb{R}^{2 n}$,

$$
\begin{aligned}
\sum_{k \in \mathbb{N}}\left(2+A+d_{k}(x, \xi)\right)^{-s} & \leq C(2+A)^{(Q+1) n-s}, \\
\sum_{k \in \mathbb{N}}\left(2+A+d_{\nu k}\right)^{-s} & \leq C(2+A)^{(Q+1) n-s} .
\end{aligned}
$$

Proposition A.9 Let $\left\{f_{k}\right\}_{k \in \mathbb{N}},\left\{g_{k}\right\}_{k \in \mathbb{N}}$ be two bounded sequences in $S\left(h_{g}^{r}, g\right)$ and in $S\left(h_{g}^{t}, g\right)$, respectively, for some $r, t \in \mathbb{R}$. Assume that

1) $\operatorname{supp} f_{k} \cap \operatorname{supp} g_{k}=\emptyset$ for every $k \in \mathbb{N}$;

2) one of the following conditions holds: either $\operatorname{supp} f_{k} \subseteq \frac{7}{4} B_{k}$ for every $k \in \mathbb{N}$ or $\operatorname{supp} g_{k} \subseteq \frac{7}{4} B_{k}$ for every $k \in \mathbb{N}$.

Setting $l_{k}=f_{k} \sharp g_{k}$, one has

$$
\sum_{k \in \mathbb{N}} \bar{l}_{k} \sharp l_{k} \in S(1, g) \quad \text { and } \quad \sum_{k \in \mathbb{N}} l_{k}^{w *} l_{k}^{w} u=\left(\sum_{k \in \mathbb{N}} \bar{l}_{k} \sharp l_{k}\right)^{w} u \text { for all } u \in \mathcal{S}\left(\mathbb{R}^{n}\right) \text {. }
$$

Furthermore, $\sum_{k \in \mathbb{N}} l_{k}^{w *} l_{k}^{w}$ can be extended to a linear continuous operator from $L^{2}\left(\mathbb{R}^{n}\right)$ to $L^{2}\left(\mathbb{R}^{n}\right)$.

Proof. We can assume that $\operatorname{supp} f_{k} \subseteq(7 / 4) B_{k}$ for every $k \in \mathbb{N}$ because the arguments below work analogously in the other case. In view of Lemma A.6, it is enough to show that

$$
\sum_{k \in \mathbb{N}}\left|\bar{l}_{k} \sharp l_{k}\right|_{j}^{g}(x, \xi) \leq C_{j}, \quad \forall(x, \xi) \in \mathbb{R}^{2 n} .
$$

We start by proving that, for any real constant $s>(Q+1) n$ and any integer $j$ one has

$$
\left|l_{k}\right|_{j}^{g}(x, \xi) \leq c\left(2+d_{\varrho}(x, \xi)\right)^{(Q+1) n-s}, \quad \forall(x, \xi) \in \mathbb{R}^{2 n},
$$

where $Q$ is the constant appearing in (A.6). This estimate follows from Lemma A.7, but it is not straightforward since the symbols $f_{k}(x, \xi)$ and 
$g_{k}(x, \xi)$ are not both supported in the rectangle $(7 / 4) B_{k}$. In view of that, we use the partition of unity given in Proposition A.4. Set

$$
g_{k \nu}(x, \xi)=\varphi_{\nu}(x, \xi)^{2} g_{k}(x, \xi) \text { for any } k, \nu \in \mathbb{N} .
$$

From Leibniz formula, we have, for any $j \in \mathbb{N}$,

$$
h_{g}(x, \xi)^{-t}\left|g_{k \nu}\right|_{j}^{g}(x, \xi) \leq C_{j}, \quad \forall \nu, k \in \mathbb{N}, \forall(x, \xi) \in \mathbb{R}^{2 n} .
$$

Since $f_{k}$ and $g_{k \nu}$ have disjoint supports, one gets $T_{N}\left(f_{k}, g_{k \nu}\right)=0$ for every $N$. Hence Lemma A.7 yields, on choosing $N \in \mathbb{N}, N+r+t \geq 0$,

$$
\begin{aligned}
& \left|f_{k \sharp} \sharp g_{k \nu}\right|_{k}^{g}(x, \xi) \leq c_{1} h_{g}(x, \xi)^{r+t+N}\left(2+d_{k}(x, \xi)+d_{\nu}(x, \xi)\right)^{-s}\left\|f_{k}\right\|_{\left(l, h_{g}^{r}\right)}\left\|g_{k \nu}\right\|_{\left(l, h_{g}^{t}\right)} \\
& \text { (A.21) } \leq c_{2}\left(2+d_{k}(x, \xi)+d_{\nu}(x, \xi)\right)^{-s}, \quad \forall(x, \xi) \in \mathbb{R}^{2 n} .
\end{aligned}
$$

In view of the finite overlapping property of $\left\{B_{\nu}\right\}_{\nu \in \mathbb{N}}$ (see Proposition A.2) and of (A.20), the sequence $\left\{\sum_{\nu \leq N} g_{k \nu}\right\}_{N \in \mathbb{N}}$ is bounded in $S\left(h_{g}^{t}, g\right)$ and converges in $C^{\infty}\left(\mathbb{R}^{n} \times \mathbb{R}^{n}\right)$ to $g_{k}$. Therefore, from the weak continuity of the map $\sharp$, it follows that the sequence $\left\{\sum_{\nu \leq N} f_{k} \sharp g_{k \nu}\right\}_{N \in \mathbb{N}}$ converges in $C^{\infty}\left(\mathbb{R}^{n} \times \mathbb{R}^{n}\right)$ to $f_{k} \sharp g_{k}$ as $N$ tends to $+\infty$. Hence by (A.21) one has

$$
\left|f_{k} \sharp g_{k}\right|_{k}^{g}(x, \xi) \leq \sum_{\nu \in \mathbb{N}}\left|f_{k} \sharp g_{k \nu}\right|_{k}^{g}(x, \xi) \leq c_{2} \sum_{\nu \in \mathbb{N}}\left(2+d_{k}(x, \xi)+d_{\nu}(x, \xi)\right)^{-s} .
$$

By (A.17) we finally get, for any $s>(Q+1) n$,

$$
\left|l_{k}\right|_{j}^{g}(x, \xi)=\left|f_{k} \sharp g_{k}\right|_{j}^{g}(x, \xi) \leq c_{3}\left(2+d_{k}(x, \xi)\right)^{(Q+1) n-s}, \quad \forall(x, \xi) \in \mathbb{R}^{2 n} .
$$

In order to prove (A.19), we want to apply Lemma A.7 to the symbols $\bar{l}_{k}, l_{k}$, thus we have to localize them in the rectangles $\left\{\frac{7}{4} B_{\nu}\right\}_{\nu \in \mathbb{N}}$. From Leibniz formula and from (A.22), it follows, for any $j \in \mathbb{N}$ and any $s>(Q+1) n$,

$$
\left|\varphi_{\nu}^{2} l_{k}\right|_{j}^{g}(x, \xi) \leq c_{4}\left(2+d_{\nu k}\right)^{(Q+1) n-s}, \quad\left|\varphi_{\mu}^{2} \bar{l}_{k}\right|_{j}^{g}(x, \xi) \leq c_{5}\left(2+d_{\mu k}\right)^{(Q+1) n-s} \text {. }
$$

An application of Lemma A.7 yields, for any $s>(Q+1) n$,

$$
\begin{aligned}
& \left|\left(\varphi_{\nu}^{2} \bar{l}_{k}\right) \sharp\left(\varphi_{\mu}^{2} l_{k}\right)\right|_{j}^{g}(x, \xi) \leq \\
& \quad \leq c_{6}\left(2+d_{\nu}(x, \xi)+d_{\mu}(x, \xi)\right)^{-s}\left(2+d_{\nu k}\right)^{(Q+1) n-s}\left(2+d_{\mu k}\right)^{(Q+1) n-s} .
\end{aligned}
$$

Furthermore, an easy check shows that the sequences $\left\{\sum_{\nu \leq N} \varphi_{\nu}^{2} l_{k}\right\}_{N \in \mathbb{N}}$, $\left\{\sum_{\mu \leq N} \varphi_{\mu}^{2} \bar{l}_{k}\right\}_{N \in \mathbb{N}}$ are bounded in $S(1, g)$ and that

$$
\sum_{\nu \leq N} \varphi_{\nu}^{2} l_{k} \frac{C^{\infty}\left(\mathbb{R}^{2 n}\right)}{N \rightarrow+\infty} l_{k}, \quad \sum_{\mu \leq N} \varphi_{\mu}^{2} \bar{l}_{k} \frac{C^{\infty}\left(\mathbb{R}^{2 n}\right)}{N \rightarrow+\infty} \bar{l}_{k}
$$


whence the weak continuity of $\sharp$ gives

$$
\sum_{\nu, \mu \leq N}\left(\varphi_{\nu}^{2} \bar{l}_{k}\right) \sharp\left(\varphi_{\mu}^{2} l_{k}\right) \underset{N \rightarrow+\infty}{\stackrel{C^{\infty}\left(\mathbb{R}^{2 n}\right)}{\longrightarrow}} \bar{l}_{k} \sharp l_{k} .
$$

This result and (A.23) finally yield

$$
\begin{aligned}
& \left|\bar{l}_{k} \sharp l_{k}\right|_{j}^{g}(x, \xi) \leq \sum_{\nu, \mu \in \mathbb{N}}\left|\left(\varphi_{\nu}^{2} \bar{l}_{k}\right) \sharp\left(\varphi_{\mu}^{2} l_{k}\right)\right|_{j}^{g}(x, \xi) \\
& \quad \leq c_{6} \sum_{\nu, \mu \in \mathbb{N}}\left(2+d_{\nu}(x, \xi)+d_{\mu}(x, \xi)\right)^{-s}\left(2+d_{\nu k}\right)^{(Q+1) n-s}\left(2+d_{\mu k}\right)^{(Q+1) n-s},
\end{aligned}
$$

and a repeated application of Lemma A.8 gives then, choosing $s>2 n(Q+1)$,

$$
\begin{aligned}
& \sum_{k \in \mathbb{N}}\left|\bar{l}_{k} \sharp l_{k}\right|_{j}^{g}(x, \xi) \leq \\
& \leq c_{6} \sum_{\nu, \mu \in \mathbb{N}}\left(2+d_{\nu}(x, \xi)+d_{\mu}(x, \xi)\right)^{-s}\left[\sum_{k \in \mathbb{N}}\left(2+d_{\nu k}\right)^{(Q+1) n-s}\left(2+d_{\mu k}\right)^{(Q+1) n-s}\right] \\
& \leq c_{7} \sum_{\nu \in \mathbb{N}} \sum_{\mu \in \mathbb{N}}\left(2+d_{\nu}(x, \xi)+d_{\mu}(x, \xi)\right)^{-s} \leq C .
\end{aligned}
$$

This proves (A.19).

The following Lemma is essentially due to D.Fujiwara [7] and to F.Hérau (see [8, Lemma 1.11]).

Lemma A.10 Let $\left\{f_{k}\right\}_{k \in \mathbb{N}},\left\{g_{k}\right\}_{k \in \mathbb{N}}$ be bounded sequences of real functions in $S\left(h_{g}^{-2}, g\right)$ and in $S(1, g)$, respectively. Let $J$ be an arbitrary (finite or infinite) subset of $\mathbb{N}$. The following holds:

1) if $\operatorname{supp} f_{k} \subseteq(7 / 4) B_{k}$, supp $g_{k} \subseteq(7 / 4) B_{k}$ for every $k \in \mathbb{N}$. Then $\sum_{k \in J} g_{k}^{2} f_{k} \in S\left(h_{g}^{-2}, g\right)$ and

$$
\sum_{k \in J} g_{k}^{w} f_{k}^{w} g_{k}^{w} u=\left(\sum_{k \in J} g_{k}^{2} f_{k}\right)^{w} u+R_{J} u, \quad \forall u \in \mathcal{S}\left(\mathbb{R}^{n}\right),
$$

where $R_{J}$ is a linear continuous operator from $L^{2}\left(\mathbb{R}^{n}\right)$ to $L^{2}\left(\mathbb{R}^{n}\right)$ with norm independent of the subset $J$.

2) if $f_{k} \geq 0$ with $\operatorname{supp} f_{k} \subseteq(7 / 4) B_{k}$ and $\operatorname{supp} g_{k} \subseteq(9 / 8) B_{k}$ for every $k \in \mathbb{N}$, there exist positive constants $C, C^{\prime}$ independent of $J$ such that (A.25) $\sum_{k \in J}\left\|\left[g_{k}^{w}, f_{k}^{w}\right] u\right\|_{0}^{2} \leq C \sum_{k \in J}\left(f_{k}^{w} \psi_{k}^{w} u, \psi_{k}^{w} u\right)+C^{\prime}\|u\|_{0}^{2}, \quad \forall u \in \mathcal{S}\left(\mathbb{R}^{n}\right)$, where $\left\{\psi_{k}\right\}_{k \in \mathbb{N}}$ is the family of functions defined in Lemma A.4. Moreover, if the seminorms of the functions $f_{k}$ and $g_{k}$ don't depend on $R$, the constant $C$ can be chosen not depending on $R$. 
We complete this appendix by proving Theorem A.5.

Proof of Theorem A.5. Inequality (A.13) is an immediate consequence of Lemma A.6 and Lemma A.7.

As for $(\mathrm{A} .14)$, set $p_{k}(x, \xi)=\left(p \psi_{k}\right)(x, \xi)$ for any $k \in \mathbb{N}$. Thus by (A.24) there exists an operator $R \in \mathcal{L}\left(L^{2}\left(\mathbb{R}^{n}\right), L^{2}\left(\mathbb{R}^{n}\right)\right)$ such that

$$
\sum_{k \in \mathbb{N}} \varphi_{k}^{w} p_{k}^{w} \varphi_{k}^{w} u=\left(\sum_{k \in \mathbb{N}} \varphi_{k}^{2} p\right)^{w} u+R u=p^{w} u+R u, \quad \forall u \in \mathcal{S}\left(\mathbb{R}^{n}\right),
$$

whence

$$
\left\|p^{w} u\right\|_{0}^{2}=\sum_{k \in \mathbb{N}}\left(p_{k}^{w} \varphi_{k}^{w} u, \varphi_{k}^{w} p^{w} u\right)_{L^{2}}-\left(R u, p^{w} u\right)_{L^{2}}, \quad \forall u \in \mathcal{S}\left(\mathbb{R}^{n}\right) .
$$

By the Cauchy-Schwarz inequality and (A.13) one gets, for an arbitrary constant $\epsilon>0$,

$$
\left\|p^{w} u\right\|_{0}^{2} \leq \frac{1}{\epsilon^{2}} \sum_{k \in \mathbb{N}}\left\|p_{k}^{w} \varphi_{k}^{w} u\right\|_{0}^{2}+\left(1+c_{1}\right) \epsilon^{2}\left\|p^{w} u\right\|_{0}^{2}+\frac{c}{\epsilon^{2}}\|u\|_{0}^{2}, \quad \forall u \in \mathcal{S}\left(\mathbb{R}^{n}\right) .
$$

By choosing $\epsilon^{2}<\left(1+c_{1}\right)^{-1}$, (A.14) readily follows.

It remains to prove (A.15). We start by observing that

$$
\left\|\varphi_{k}^{w} p_{k}^{w} u\right\|_{0}^{2} \leq 2\left\|\varphi_{k}^{w} p^{w} u\right\|_{0}^{2}+2\left\|\varphi_{k}^{w}\left(\left(1-\psi_{k}\right) p\right)^{w} u\right\|_{0}^{2} .
$$

An application of Proposition A.9 with $f_{k}=\varphi_{k}$ and $g_{k}=\left(1-\psi_{k}\right) p$ yields

$$
\sum_{k \in \mathbb{N}}\left\|\varphi_{k}^{w} p_{k}^{w} u\right\|_{0}^{2} \leq 2 \sum_{k \in \mathbb{N}}\left\|\varphi_{k}^{w} p^{w} u\right\|_{0}^{2}+C_{1}\|u\|_{0}^{2}, \quad \forall u \in \mathcal{S}\left(\mathbb{R}^{n}\right) .
$$

Hence, from (A.13) it follows

$$
\sum_{k \in \mathbb{N}}\left\|\varphi_{k}^{w} p_{k}^{w} u\right\|_{0}^{2} \leq C_{2}\left\|p^{w} u\right\|_{0}^{2}+C_{2}^{\prime}\|u\|_{0}^{2}, \quad \forall u \in \mathcal{S}\left(\mathbb{R}^{n}\right)
$$

with $C_{2}$ independent of $R$.

Since $p_{k}^{w} \varphi_{k}^{w}=\varphi_{k}^{w} p_{k}^{w}+\left[p_{k}^{w}, \varphi_{k}^{w}\right]$ one has, for every $u \in \mathcal{S}\left(\mathbb{R}^{n}\right)$,

$$
\sum_{k \in \mathbb{N}}\left\|\varphi_{k}^{w} p_{k}^{w} u\right\|_{0}^{2} \geq \frac{1}{2} \sum_{k \in \mathbb{N}}\left\|p_{k}^{w} \varphi_{k}^{w} u\right\|_{0}^{2}-\sum_{k \in \mathbb{N}}\left\|\left[p_{k}^{w}, \varphi_{k}^{w}\right] u\right\|_{0}^{2}
$$

so that (A.15) is proved if we show that

$$
\sum_{k \in \mathbb{N}}\left\|\left[p_{k}^{w}, \varphi_{k}^{w}\right] u\right\|_{0}^{2} \leq C_{3}\left\|p^{w} u\right\|_{0}^{2}+C_{3}^{\prime}\|u\|_{0}^{2}, \quad \forall u \in \mathcal{S}\left(\mathbb{R}^{n}\right) .
$$


Let us now set $\bar{N}:=\left\{k \in \mathbb{N}:\left|\xi_{k}\right| \geq R\right\}$ and note that $p_{k} \in S(1, g)$, uniformly in $k$, when $k \notin \bar{N}$. Hence we can apply (A.25) with $f_{k}=p_{k}$ and $g_{k}=\varphi_{k}$ (here we also use (A.12)) and get

$$
\sum_{k \in \mathbb{N}}\left\|\left[p_{k}^{w}, \varphi_{k}^{w}\right] u\right\|_{0}^{2} \leq C_{4} \sum_{k \in \bar{N}}\left(p_{k}^{w} \psi_{k}^{w} u, \psi_{k}^{w} u\right)_{L^{2}}+C_{4}^{\prime}\|u\|_{0}^{2}, \quad \forall u \in \mathcal{S}\left(\mathbb{R}^{n}\right)
$$

Observe that $C_{4}$ is independent of $R$ when $\delta_{k}^{-2+|\alpha|} \epsilon_{k}^{-2+|\beta|}\left|\partial_{x}^{\alpha} \partial_{\xi}^{\beta} p_{\mid B_{k}}\right|$ are uniformly bounded in $R$. Therefore, by (A.24), one has

$$
\sum_{k \in \mathbb{N}}\left\|\left[p_{k}^{w}, \varphi_{k}^{w}\right] u\right\|_{0}^{2} \leq C_{5}\left(\left(\sum_{k \in \bar{N}} \psi_{k}^{3} p\right)^{w} u, u\right)_{L^{2}}+C_{5}^{\prime}\|u\|_{0}^{2}, \quad \forall u \in \mathcal{S}\left(\mathbb{R}^{n}\right)
$$

An application of the Fefferman-Phong inequality gives

$$
\left(\left(\sum_{k \in \bar{N}} \psi_{k}^{3} p\right)^{w} u, u\right)_{L^{2}} \leq C_{6}\left(p^{w} u, u\right)+C_{6}^{\prime}\|u\|_{0}^{2}, \quad \forall u \in \mathcal{S}\left(\mathbb{R}^{n}\right) .
$$

This estimate together with (A.27) yields (A.26). The proof of (A.15) is complete.

\section{References}

[1] Beals, R.: Weighted distribution spaces and pseudodifferential operators. J. Analyse Math. 39 (1981), 131-187.

[2] Beals, R. and Fefferman, C. L.: On local solvability of linear partial differential equations. Ann. of Math. (2) 97 (1973), 482-498.

[3] Boutet de Monvel, L., Grigis, A. and Helffer, B.: Parametrixes d'opérateurs pseudo-différentiels à caractéristiques multiples. In Journées: Équations aux Dérivées Partielles de Rennes (1975), 93-121. Astérisque 34-35, Soc. Math. France, Paris, 1976.

[4] Fefferman, C. L. And Phong, D. H.: On positivity of pseudodifferential operators. Proc. Nat. Acad. Sci. U.S.A. 75 (1978), 4673-4674.

[5] Fefferman, C. L.: The uncertainty principle. Bull. Amer. Math. Soc. (N.S.) 9 (1983), 129-206.

[6] Fefferman, C. L.: The SAK Principle. Lectures given in Turin, Bimestre INDAM 2003.

[7] Fujiwara, D.: A construction of approximate positive parts of essentially selfadjoint pseudodifferential operators. Comm. Pure Appl. Math. 37 (1984), 101-147.

[8] HÉrau, F.: Fefferman's SAK principle in one dimension. Ann. Inst. Fourier (Grenoble) $\mathbf{5 0}$ (2000), no. 4, 1229-1264. 
[9] Hörmander, L.: The Weyl Calculus of pseudodifferential operators. Comm. Pure Appl. Math. 32 (1979), 360-444.

[10] Hörmander, L.: The analysis of linear partial differential operators. Vol. I and Vol. III. Fundamental Principles of Mathematical Sciences 256 and 274. Springer-Verlag, Berlin, 1983 and 1985.

[11] Lerner, N. And Nourrigat, J.: Lower bounds for pseudo-differential operators. Ann. Inst. Fourier (Grenoble) 40 (1990), no. 3, 657-682.

[12] Maniccia, L. And Mughetti, M.: Parametrix construction for a class of anisotropic operators. Ann. Univ. Ferrara Sez. VII (N.S.) 49 (2003), 263-284.

[13] Parmeggiani, A.: Subunit balls for symbols of pseudodifferential operators. Adv. Math. 131 (1997), no. 2, 357-452.

[14] Parmeggiani, A.: A crash introduction to pseudodifferential calculus. In Geometry Seminars (Bologna, 1999/2000), 183-223, Univ. Stud. Bologna, Bologna, 2001.

[15] Parmeggiani, A.: Lectures in Reims. Unpublished notes, 2000.

[16] Tataru, D.: On the Fefferman-Phong inequality and related problems. Comm. Partial Differential Equations 27 (2002), no. 11-12, 2101-2138.

[17] Treves, F.: Topological vector spaces, distributions and kernels. Academic Press, New York-London, 1967.

Recibido: 13 de octubre de 2003

Lidia Maniccia

Department of Mathematics

University of Bologna

Piazza di Porta S. Donato 5

40127 Bologna, Italy

maniccia@dm. unibo.it

Marco Mughetti

Department of Mathematics

University of Bologna

Piazza di Porta S. Donato 5

40127 Bologna, Italy

mughetti@dm. unibo. it 